\title{
Genomic insights into the evolution of Echinochloa species as weed and orphan crop
}

\author{
Dongya Wu 10 1,18, Enhui Shen 1,2,18, Bowen Jiang ${ }^{1}$, Yu Feng ${ }^{3,4}$, Wei Tang ${ }^{5}$, Sangting Lao ${ }^{1}$, Lei Jia ${ }^{1}$, Han-Yang Lin ${ }^{3}$,

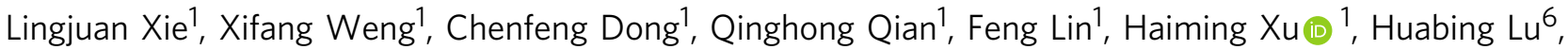 \\ Luan Cutti (1) ${ }^{7}$, Huajun Chen ${ }^{8}$, Shuiguang Deng ${ }^{8}$, Longbiao Guo (1) ${ }^{5}$, Tse-Seng Chuah ${ }^{9}$, Beng-Kah Song ${ }^{10}$, \\ Laura Scarabel $^{11}$, Jie Qiu (1) ${ }^{12}$, Qian-Hao Zhu ${ }^{13}$, Qin Yu (1) ${ }^{14}$, Michael P. Timko ${ }^{15}$, Hirofumi Yamaguchi ${ }^{16}$, \\ Aldo Merotto $\mathrm{Jr}^{7}$, Yingxiong Qiu ${ }^{3}$, Kenneth M. Olsen (1) ${ }^{17}$, Longjiang Fan ${ }^{1,2} \&$ Chu-Yu Ye (D) ${ }^{1 凶}$
}

As one of the great survivors of the plant kingdom, barnyard grasses (Echinochloa spp.) are the most noxious and common weeds in paddy ecosystems. Meanwhile, at least two Echinochloa species have been domesticated and cultivated as millets. In order to better understand the genomic forces driving the evolution of Echinochloa species toward weed and crop characteristics, we assemble genomes of three Echinochloa species (allohexaploid E. crus-galli and E. colona, and allotetraploid E. oryzicola) and re-sequence 737 accessions of barnyard grasses and millets from 16 rice-producing countries. Phylogenomic and comparative genomic analyses reveal the complex and reticulate evolution in the speciation of Echinochloa polyploids and provide evidence of constrained disease-related gene copy numbers in Echinochloa. A population-level investigation uncovers deep population differentiation for local adaptation, multiple target-site herbicide resistance mutations of barnyard grasses, and limited domestication of barnyard millets. Our results provide genomic insights into the dual roles of Echinochloa species as weeds and crops as well as essential resources for studying plant polyploidization, adaptation, precision weed control and millet improvements.

\footnotetext{
${ }^{1}$ Institute of Crop Science \& Institute of Bioinformatics, Zhejiang University, Hangzhou 310058, China. ${ }^{2}$ Zhejiang University Zhongyuan Institute, Zhengzhou 450000, China. ${ }^{3}$ Institute of Ecology, Zhejiang University, Hangzhou 310058, China. ${ }^{4}$ Chengdu Institute of Biology, Chinese Academy of Sciences, Chengdu 610041, China. ${ }^{5}$ State Key Laboratory of Rice Biology, China National Rice Research Institute, Hangzhou 310006, China. ${ }^{6}$ Institute of Maize and Upland Grain, Zhejiang Academy of Agricultural Sciences, Dongyang 322105, China. ${ }^{7}$ Department of Crop Sciences, Agricultural School, Federal University of Rio Grande do Sul, Porto Alegre, RS 91540-000, Brazil. ${ }^{8}$ College of Computer Science and Technology, Zhejiang University, Hangzhou 310058, China. ${ }^{9}$ Faculty of Plantation and Agrotechnology, Universiti Teknologi MARA, 02600 Arau, Perlis, Malaysia. ${ }^{10}$ School of Science, Monash University Malaysia, 46150 Bandar Sunway, Selangor, Malaysia. ${ }^{11}$ Istituto per la Protezione Sostenibile delle Piante (IPSP), CNR, Viale dell'Università, 16, 35020 Legnaro (PD), Italy. ${ }^{12}$ Shanghai Key Laboratory of Plant Molecular Sciences, College of Life Sciences, Shanghai Normal University, Shanghai 200235, China. ${ }^{13}$ CSIRO Agriculture and Food, GPO Box 1700 Canberra, ACT 2601, Australia. ${ }^{14}$ Australian Herbicide Resistance Initiative, School of Agriculture and Environment, University of Western Australia, Crawley, WA 6009, Australia. ${ }^{15}$ Department of Biology, University of Virginia, Charlottesville, VA 22904, USA. ${ }^{16}$ Osaka Prefecture University, Sakai, Osaka 599-8531, Japan. ${ }^{17}$ Department of Biology, Washington University in St. Louis, St. Louis, MO 63130, USA. ${ }^{18}$ These authors contributed equally: Dongya Wu, Enhui Shen. $\aleph_{\text {email: yecy@zju.edu.cn }}$
} 
W eeds are among the super survivors of the plant kingdom as they generally have large populations and wide geographic distributions ${ }^{1}$. Barnyard grasses (Echinochloa P. Beauv.) are among the most problematic and dominant weeds in world agricultural systems, especially in rice paddy fields ${ }^{2}$. Echinochloa species are distributed globally from tropical to temperate regions and across a wide range of habitats, including agricultural and non-agricultural fields. Barnyard grasses in agroecosystems exhibit typical weed adaptation syndromes, such as aggressive competition with high nitrogen utilization, crop mimicry, allelopathy, and abiotic stress resistance (including herbicide resistance) $^{3-7}$. The biosynthesis and release of allelochemicals (e.g., benzoxazinoid and momilactone) allow barnyard grasses to suppress the growth of surrounding plants (including crops) and to resist pathogen attack ${ }^{8,9}$. The tillering and grain yield of rice can be reduced by up to $50-70 \%$ because of the competition pressure of barnyard grasses 5,10 . Herbicides are widely used for the control of barnyard grasses, which has resulted in resistance evolution to herbicides of multiple sites-of-action in many barnyard grass populations across the world ${ }^{11-14}$. These mainly include resistance to the auxin mimic quinclorac and inhibitors of acetolactate synthase (ALS) (e.g., penoxsulam and bispyribac-sodium), acetyl-CoA carboxylase (ACCase) (e.g., fenoxaprop-ethyl and cyhaloprop-butyl), 5-enolpyruvylshikimate3-phosphate synthase (EPSPS) (e.g., glyphosate), photosystem II (PSII; e.g., atrazine and propanil), and very-long-chain fatty acid synthase (e.g., butachlor) ${ }^{13}$. Global resistance evolution to major herbicides in barnyard grass populations threatens the sustainability of herbicide technology and, thus, of rice production. Overall, barnyard grasses are excellent resources or systems for understanding plant tolerance to stress, due to their high adaptability to artificial and wild environments.

Owing to their highly plastic plant architecture and morphological similarities across the genus, barnyard grass species are very difficult to delimit on the basis of morphological criteria. Consequently, species names are often used indiscriminately without standard identification criteria, leading to confusion in global species distribution patterns and impeding local precision weed control. Among $\sim 50$ species of the genus Echinochloa ${ }^{15}, E$. crus-galli (L.) P. Beauv. $(2 \mathrm{n}=6 \times=54)$, E. colona (L.) Link $(2 \mathrm{n}=6 \mathrm{x}=54)$, and $E$. oryzicola Vasinger $(2 \mathrm{n}=4 \times=36)$ [synonym E. phyllopogon (Stapf) Stapf ex Kossenko] are the three most widespread species in agroecosystems ${ }^{2}$. Echinochloa oryzicola is assumed to be an ancestral parent of E. crus-galli ${ }^{16}$.

At least two barnyard millet species have been domesticated and cultivated as crops ${ }^{17}$, E. crus-galli var. esculenta [synonym E. utilis Ohwi \& Yabuno or E. esculenta (A. Braun) H. Scholz] and E. colona var. frumentacea (Link) Ridl. (synonym E. frumentacea Link) ${ }^{7,18-20}$ (Supplementary Note 1). The domestication of $E$. crus-galli var. esculenta has been estimated to have occurred $\sim 5000$ years ago, and it is cultivated mainly in the cool and dry areas of East Asia where rice does not grow well ${ }^{21}$. Echinochloa colona var. frumentacea is cultivated as a cereal crop called 'jungle rice', mainly in the Indian subcontinent ${ }^{7,18}$. Despite the good nutritional and agronomic value of barnyard millets ${ }^{7}$, little is known about the processes and genetic basis of their domestication.

To date, only the genome of the diploid species E. haploclada (Stapf) Stapf $(2 n=2 \times=18)$, which is a closely related species of maternal donor of E. crus-galli, has been sequenced and assembled to the chromosome level in the Echinochloa genus ${ }^{10}$. To better understand the evolution and adaptation of Echinochloa as weeds and to gain insights into the domestication of barnyard millets as crops, we sequence and assemble the genomes of three prevalent Echinochloa species (E. crus-galli, E. colona, and E. oryzicola) using diploid-assisted scaffolding method DipHic and perform a large-scale genomic investigation using a global panel of 737 barnyard grasses and millets from 16 countries covering rice production areas worldwide. In addition, we assess the genomic characteristics of the Echinochloa genus that allows its species to evolve as problematic weeds and orphan crops.

\section{Results}

Assembly of Echinochloa reference genomes. We sequenced one domesticated millet accession (E. colona var. frumentacea) and two weedy accessions (E. crus-galli and E. oryzicola) to generate genomes for the three Echinochloa species.

Echinochloa colona is an allohexaploid species, and the domesticated millet accession E. colona var. frumentacea, PI463783, was selected as a candidate representative of the $E$. colona genome. Var. frumentacea is cross-compatible with other varieties, such as the weedy var. colon $a^{21}$. The estimated genome size of var. frumentacea (PI463783), based on the $k$-mer survey using Illumina short reads, was $\sim 1.18 \mathrm{~Gb}$, which was consistent with its $\mathrm{C}$-value of $1.30 \mathrm{pg} / 1 \mathrm{C}^{22}$. Its heterozygosity level was estimated to be $0.131 \%$. Using $48 \times$ coverage $(57.2 \mathrm{~Gb}$ ) of PacBio $\mathrm{HiFi}$ reads with an average read length of $15.1 \mathrm{~kb}$, a de novo assembly yielded a genome of $1.13 \mathrm{~Gb}$ with a contig N50 size of $24.8 \mathrm{Mb}$. The assembly was further refined using chromosome contact maps with $\sim 100 \times \mathrm{Hi}-\mathrm{C}$ data. Owing to the nature of hexaploidy, the sequence similarity among the three subgenomes severely reduced the accuracy of the chromosome scaffolding using regular Hi-C-assisted methods (Supplementary Fig. 1a). Therefore, we developed a diploid-assisted scaffolding method (named as DipHiC) to scaffold the genomes of polyploid Echinochloa species (Fig. 1a; See details at Supplementary Note 2). DipHiC includes two steps, subgenome distinguishing and chromosome building. To assign contigs to the three subgenomes of E. colona, we integrated the genetic relationship to diploid species, allelic contig information, and inter-contig interactions via multiple rounds of clustering and correction (Supplementary Fig. 2). Consequently, $97.1 \%$ of contig sequences were assigned into the three subgenomes, which were designated $\mathrm{DH}, \mathrm{EH}$, and $\mathrm{FH}(\mathrm{AH}, \mathrm{BH}$, and $\mathrm{CH}$ have been designated for the three subgenomes of $E$. crus-galli, with ' $\mathrm{H}$ ' referring to hexaploid) (Supplementary Note 2). In each subgenome, the directional interactions and chromosome pre-assignment with the diploid reference (E. haploclada) were utilized to construct linkages among contigs. After correction and ordering, we finally assembled the genome of $E$. colona with subgenome sizes of $436.4(\mathrm{DH}), 310.5(\mathrm{EH})$, and $347.1 \mathrm{Mb}(\mathrm{FH})$ (Fig. 1b; Table 1; Supplementary Fig. 1b).

The quality of the assembly was validated through the mapping of $90.4 \%$ of the transcriptomic reads obtained by RNA-Seq and $97.9 \%$ of the genomic short reads obtained by Illumina sequencing to the assembly. In addition, $97.0 \%$ of the 4896 core conserved Poales genes (BUSCO) were completely recovered, and the long terminal repeat (LTR) assembly index (LAI) score was 22.5 (where $>20$ indicates a gold quality). Clustering of the counts of 13-mer sequences that differentiate homoeologous chromosomes enables consistent partitioning of the genome into subgenomes (Supplementary Fig. 3). Centromeric regions could be found in all chromosomes containing a 155-bp tandem repeat, similar to other grass species ${ }^{23}$, and telomeric regions could be determined in most chromosomes, including 10 two-end chromosomes and 15 single-end chromosomes. High genomic synteny was observed between the E. colona assembly and the diploid and outgroup genomes (Setaria italica and Oryza sativa) (Supplementary Fig. 4a). Cumulatively, these results underscore the reliability of the E. colona assembly.

The draft genomes (contig-level) of hexaploid E. crus-galli (STB08) and tetraploid E. oryzicola (ZJU2) have been assembled 


\section{a}

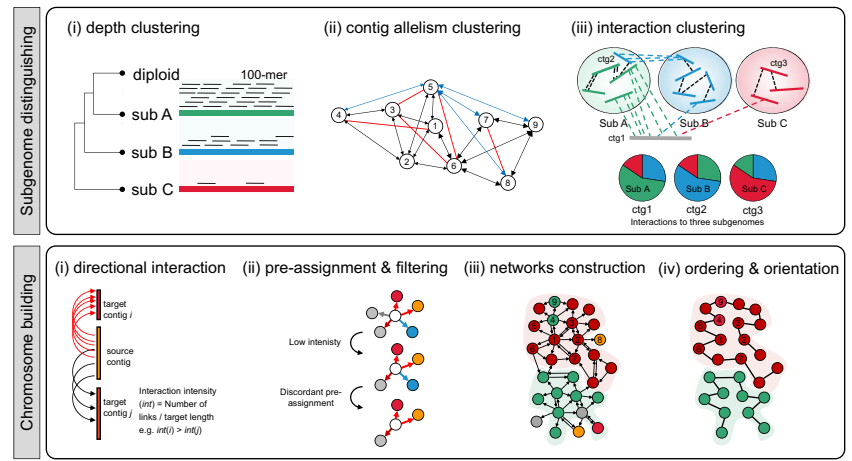

b

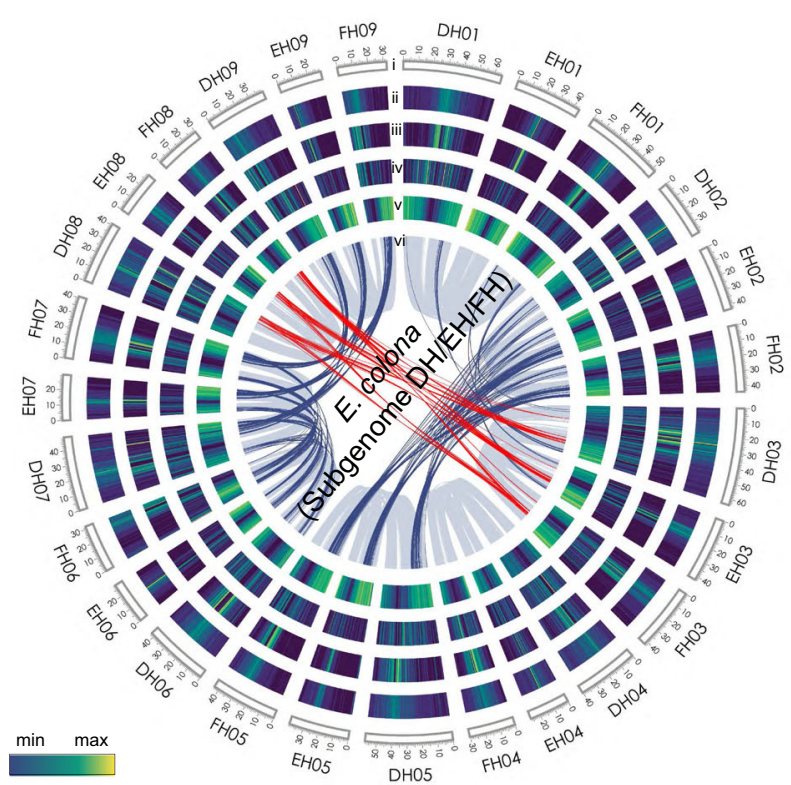

C
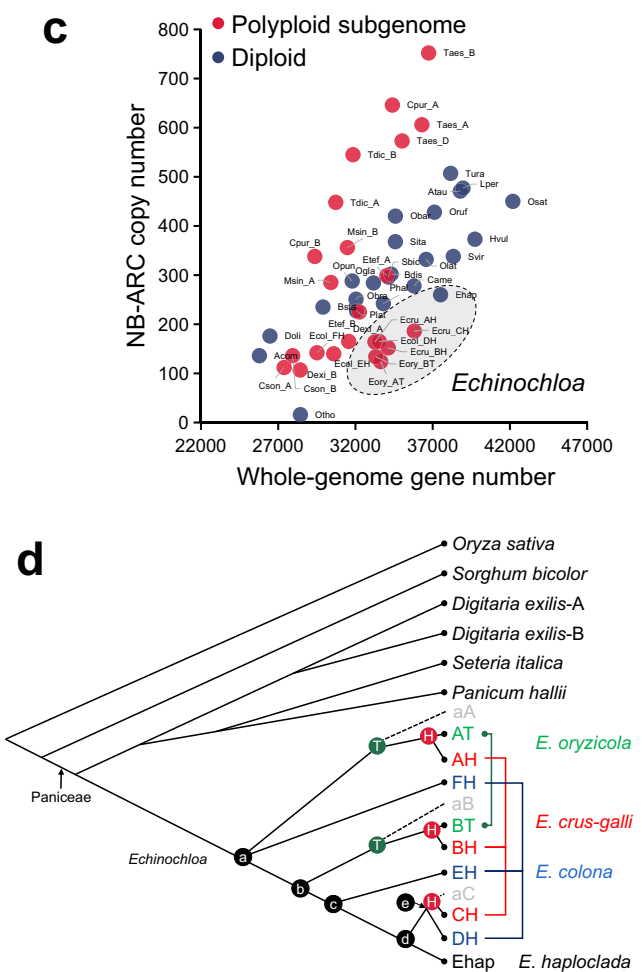

e

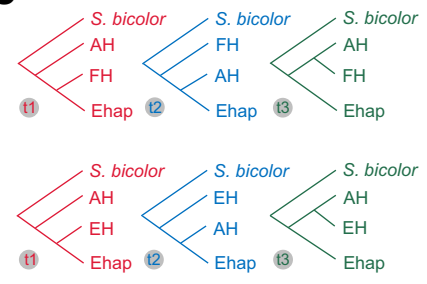

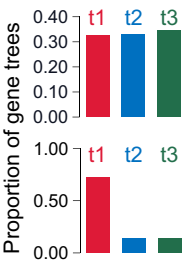

Fig. 1 Assemblies of reference genomes and speciation of the Echinochloa genus. a Overview of the diploid-assisted scaffolding method (DipHiC) workflow in hexaploid assembly. Two modules, subgenome distinguishing and chromosome building, are implemented in DipHiC. Depth information from mapping the 100-mers generated from the diploid assembly to the hexaploid draft assembly, contig allelism, and inter-contig interactions are integrated in the subgenome distinguishing module. Directional interactions among contigs within single subgenome and chromosome group pre-assignment are determined using diploid assembly to anchor contigs and build chromosomes. b Features of the Echinochloa colona reference genome. The chromosomes are shown in track i. Track ii-v show the densities of repeat elements (ii), Gypsy (iii), Copia (iv), and gene density (v). Track vi shows the syntenic blocks. Gray links show syntenic relationship among homologous subgenomes. Dark-blue and red links show syntenic relationships derived from an ancient wholegenome duplication event shared by Poaceae. $\mathbf{c}$ Distribution of the NB-ARC gene family size in different Poaceae genomes. See the full names of species in Supplementary Data 1. Echinochloa (sub)genomes are highlighted in the dashed ellipse. $\mathbf{d}$ Phylogeny of Echinochloa by integrating single-copy gene trees using ASTRAL. Dashed branches represent extinction or unknown status. ' $\mathrm{T}$ ' and ' $\mathrm{H}$ ' in the green and red nodes represent tetraploidization and hexaploidization, respectively. D. exilis-A and -B, subgenomes A and B in Digitaria exilis; Ehap, Echinochloa haploclada; AT and BT, subgenomes A and B in tetraploid E. oryzicola; $\mathrm{AH}, \mathrm{BH}$, and $\mathrm{CH}$, subgenomes $\mathrm{A}, \mathrm{B}$ and $\mathrm{C}$ in hexaploid $\mathrm{E}$. crus-galli; $\mathrm{DH}, \mathrm{EH}$, and $\mathrm{FH}$, subgenomes $\mathrm{D}, \mathrm{E}$ and $\mathrm{F}$ in hexaploid $E$. colona; a and $\mathrm{aB}$, ancestral diploid genomes of subgenomes $\mathrm{A}$ and $\mathrm{B}$. Subgenomes from the same species are linked by color-coded lines. $\mathbf{e}$ The gene-tree quartet frequency around the divergence node a in the Echinochloa phylogeny. Red (t1) represents the ASTRAL and concatenation species topology; blue (t2) and green ( $\mathrm{t} 3$ ) trees represent two alternative topologies. The colored bars on the right panel show the frequencies of three topologies. Source data are provided as a Source data file.

previously ${ }^{10}$. Here, we further generated and used $\mathrm{Hi}-\mathrm{C}$ data (122.8 Gb for E. oryzicola with $\sim 117$-fold genome coverage and $181.0 \mathrm{~Gb}$ for E. crus-galli with $\sim 129$-fold coverage) to anchor the contigs into chromosomes by using DipHiC (Supplementary Figs. 5 and 6; Supplementary Note 3 and 4). In total, $97.9 \%$ and 96.2\% of sequences were anchored onto 18 and 27 chromosomes of E. oryzicola and E. crus-galli, respectively (Supplementary Figs. 7 and 8). The subgenome-specific 13-mer and transposon element analysis further supported the accuracy of subgenome distinguishing for the two polyploid genomes (Supplementary Fig. 9). In total, 502.3 and $416.7 \mathrm{Mb}$ contigs were assigned to the two E. oryzicola subgenomes $\mathrm{AT}$ and $\mathrm{BT}$ (where ' $\mathrm{T}$ ' refers to tetraploid), and three E. crus-galli subgenomes of sizes 473.2 (AH), $399.0(\mathrm{BH})$, and $416.8 \mathrm{Mb}(\mathrm{CH})$ were determined (Table 1). Centromeric regions were also found in all chromosomes (Supplementary Fig. 8b).

Among the nine subgenomes of the four Echinochloa species, including the three polyploids used in this study and the diploid E. haploclada genome (hereafter 'Ehap'), the subgenome sizes varied from $311 \mathrm{Mb}(\mathrm{EH})$ to $502 \mathrm{Mb}(\mathrm{AT})$, mostly resulting from the alterations of repeat elements (Table 1), which accounted for $36.6 \%(\mathrm{EH})$ to $53.7 \%(\mathrm{AH})$ of the subgenome size. The majority 
Table 1 Summary of the assembly and annotation of Echinochloa reference genomes.

\begin{tabular}{|c|c|c|c|c|c|}
\hline E. oryzicola & & AT & 502.3 & 33,635 & 53.5 \\
\hline \multirow[t]{3}{*}{ E. crus-galli } & $2 n=6 x=54$ & Whole genome & 1339.8 & 104,780 & 47.7 \\
\hline & & $\mathrm{AH}$ & 473.2 & 33,261 & 53.7 \\
\hline & & $\mathrm{BH}$ & 399.0 & 34,124 & 45.2 \\
\hline \multirow{3}{*}{ E. colona } & & $\mathrm{DH}$ & 436.4 & 33,538 & 50.4 \\
\hline & & $\mathrm{EH}$ & 310.5 & 30,593 & 36.6 \\
\hline & & $\mathrm{FH}$ & 347.1 & 29,510 & 43.4 \\
\hline
\end{tabular}

of repeat elements were Gypsy-type long terminal repeat (LTR) elements (Supplementary Fig. 10).

A total of $68,544,104,780$, and 93,697 genes were predicted in the E. oryzicola, E. crus-galli, and E. colona genome assemblies, respectively (Table 1). Compared with the diploid E. haploclada (37,510 genes), the polyploid subgenomes showed gene losses. Compared to other Poaceae species (Supplementary Data 1), except for Oropetium thomaeum (L.f.) Trin., which has the smallest known genome size in this family at $\sim 285 \mathrm{Mb}^{24}$; Echinochloa is characterized by marked contraction in the sizes of gene families related to biotic stress response (e.g., NB-ARC domain, legume lectin, D-mannose-binding lectin, UDPglucoronosyl and UDP-glucosyl transferase) (Fig. 1c; Supplementary Fig. 11). This is probably a result of fitness costs associated with maintaining these stress respons $\mathrm{e}^{25}$. Interestingly, across the Poaceae, the NB-ARC gene family showed a high coefficient of variation (CV) in size, followed by other diseaserelated gene families (Supplementary Fig. 12). The evolutionary dynamics of the NB-ARC gene family size has also been observed in other plants (e.g., Phaseolus) ${ }^{26}$. Additionally, the number of NB-ARC genes generally changed linearly along with the diploid genome size $\left(R^{2}=0.69\right)$. This is in contrast to the dramatic copy number variations in polyploid subgenomes, where the NB-ARC family was expanded in Triticum and contracted in Echinochloa, although the two polyploids shared similar formation processes (Fig. 1c). The NB-ARC genes were significantly enriched on chromosome 4 of Echinochloa $\left(P<1 \times 10^{-5}\right.$, Fisher's exact test), which is syntenic to chromosome 11 of rice ${ }^{27}$ (Supplementary Fig. 13). Chromosome 4 of Echinochloa and chromosome 11 of rice were also found to have the highest transposon density in both genomes. It has been speculated that the abundance of transposable elements may have contributed to the copy number dynamics of the NB-ARC gene families across the Poaceae, facilitating rapid adaptation to local environments ${ }^{28}$. In contrast, gene families related to abiotic stress response (e.g., the AP2, C2, GRAS, and MYB-like DNA-binding domain families) showed conservation in size, with relatively low CVs across the Poaceae (Supplementary Figs. 11 and 12). Nonetheless, despite apparent genomic contractions after polyploidization, several local expansions, possibly underlying specific adaptations, were observed in Echinochloa. For example, an expansion from $\mathrm{BT}$ to $\mathrm{BH}$ resulting from the massive local duplication of two genes, one encoding an O-methyltransferase that plays a key role in lignin biosynthesis, stress tolerance, and disease resistance in plants ${ }^{29}$, and the other encoding a pyridoxal-dependent decarboxylase involved in plant nitrogen metabolism ${ }^{30}$ (Supplementary Fig. 4b).

Phylogeny of the Echinochloa genus. We first calculated the synonymous substitutions per synonymous site $\left(K_{\mathrm{s}}\right)$ of syntenic gene pairs among the Echinochloa subgenomes and other genomes of evolutionarily neighboring species, including O. sativa L., Sorghum bicolor (L.) Moench, Digitaria exilis (Kippist) Stapf (separated into two subgenomes D. exilis-A and D. exilis-B), $S$. italica (L.) P. Beauv., and Panicum hallii Vasey (Supplementary Fig. 14a). The peaks of $K_{\mathrm{s}}$ distributions are regarded as divergence events. The genus-level phylogeny, which was built based on the $K_{\mathrm{s}}$ distribution, was consistent with previous phylogenetic analyses using plastomes across the entire Poaceae ${ }^{31}$ (Supplementary Fig. 14b). Assuming an average substitution rate of $6.5 \times 10^{-9}$ substitutions per year per synonymous site ${ }^{10}$, Echinochloa and Digitaria were estimated to diverge at approximately 20.0 million years ago (mya), and the divergence of Echinochloa from Setaria and Panicum occurred $\sim 14.6$ mya, in line with the previous estimation ${ }^{10}$. Within Echinochloa, the LTR insertion time estimation dated the tetraploidization event of E. oryzicola to be $\sim 2.0$ mya (Supplementary Fig. 15), and the hexaploidization time of $E$. crus-galli was $\sim 0.3$ mya according to the $K_{\mathrm{s}}$ distributions between $\mathrm{AH}(\mathrm{BH})$ and its ancestral subgenome AT (BT) (Supplementary Fig. 14). Details of the hexaploidization of E. colona could not be uncovered because of the lack of parental genomes, but subgenome DH in E. colona showed a fairly close genetic distance to $\mathrm{CH}$ in E. crus-galli, with an estimated divergence time of $\sim 0.3$ mya, overlapping with the hexaploidization time of $E$. crus-galli. This suggests that the ancestral diploid of $\mathrm{CH}$ is genetically close to the ancestral genome of $\mathrm{DH}$ and, therefore, $E$. colona is a neo-hexaploid species, just like E. crus-galli (Fig. 1d; Supplementary Fig. 14). In Echinochloa, subgenome A (including $\mathrm{AT}$ and $\mathrm{AH}$ ) diverged from the other subgenomes $~ 4.6$ mya. However, the $K_{\mathrm{s}}$ distribution peaks between subgenome $\mathrm{FH}$ and subgenome A (including AT and $\mathrm{AH}$ ) and between subgenome FH and Ehap, overlapped with the $K_{\mathrm{s}}$ peak between subgenome A and Ehap, implying radiative evolution at the first divergence in Echinochloa (Supplementary Fig. 14).

We reconstructed the maximum-likelihood (ML) phylogeny based on a concatenated matrix of 3557 single-copy orthologs among the 16 (sub)genomes and also a coalescent-based phylogeny by integrating these single-copy gene trees (Fig. 1d; Supplementary Fig. 16a, b). Consistent with the result of the $K_{\mathrm{s}}$ distribution analysis, a weak support existed at the node where $\mathrm{FH}$ diverged from the other subgenomes in the coalescent tree, confirming radiative evolution. Using single-copy orthologs, we counted the numbers of three gene topologies among $\mathrm{AH}, \mathrm{FH}$, Ehap, and an outgroup (one species tree and two incongruent trees for a triplet with an outgroup). The 1:1:1 ratio in the gene counts of the three trees implied possible incomplete lineage sorting (ILS) at the first Echinochloa divergence (Fig. 1e; Supplementary Fig. 16c). Subsequently, quantifying introgression via branch lengths (QuIBL), a method based on the internal branch length, was used to infer the proportions of potential ILS 
or introgression event contributing to topological incongruence ${ }^{32}$. The results revealed that the discordance between the gene and species trees in Echinochloa was more likely caused by ILS rather than introgression, similar to the Oryza genus ${ }^{33}$ (Supplementary Data 2). In addition, the unbiased distribution of three topologies of triplet (AH, FH, and Ehap) further supported the possibility of ILS in Echinochloa (Supplementary Fig. 17). To trace the ILS footprints along with the evolutionary process, we measured the topological discordance in various triplets of Echinochloa subgenomes (Supplementary Fig. 16d). With an increase in divergence time interval, the proportion of topological discordance decreased at the rate of $10.6 \%$ per million years at the whole-genome level in Echinochloa (Supplementary Fig. 16d). Conclusively, the case in Echinochloa confirms the substantial impact of ILS on phylogenetic inference, especially for groups that experienced rapid radiative evolution.

We noticed that Digitaria and Setaria-Panicum together formed a monophyletic group (clade) in the concatenated- and coalescent-based phylogeny, rather than a polyphyletic relationship, as inferred from the $K_{\mathrm{s}}$ distribution analysis (Supplementary Figs. $14 \mathrm{c}, 16 \mathrm{a}, \mathrm{b})$. Specifically, the $K_{\mathrm{s}}$ peak of Setaria-Echinochloa or Panicum-Echinochloa shifted to the left of Digitaria-Echinochloa (nodes 4 and 3 in Fig. 1d; Supplementary Fig. 14). To validate whether introgression caused incongruence, triplet gene trees were built (Supplementary Fig. 18). In total, only $40.03-41.64 \%$ of the gene trees supported the species tree in the triplet of Setaria, Digitaria, and Echinochloa with Oryza as an outgroup, although the time interval between the Digitaria and Setaria speciation times was greater than 5 million years, indicating that genetic exchange events (i.e., introgression), rather than ILS, drove topological incongruence. Additionally, $66.19 \%$ of gene trees matched the species topology in the triplet (Sorghum, Digitaria, and Echinochloa) with Oryza as an outgroup, smaller than the proportion of $\sim 79 \%$, estimated based on the triplet divergence interval of 3.5 million years under the condition of only ILS (Supplementary Fig. 16d). Frequent introgression among the genera was confirmed by QuIBL analyses (Supplementary Data 2). Taken together, these analyses indicate that both introgression and ILS play important roles in generating topological discordance between gene and species trees in the Paniceae tribe.

Population structure and demography of global Echinochloa species. To explore the diversity and population structure of Echinochloa on a global scale, a total of 707 accessions of barnyard grasses from rice paddy ecosystem (paddy fields and surroundings), including 328 accessions that have been sequenced in our previous study 6 and 409 newly sequenced in this study, and 30 cultivated accessions of barnyard millets from 16 countries were collected and used in genomic investigation (Fig. 2a; Supplementary Data 3). Clean reads were first mapped to the E. crusgalli (STB08) reference genome. Based on the read mapping rates and genome coverage (Fig. 2b; Supplementary Fig. 19), together with the morphological traits and genome sizes estimated by flow cytometry or $k$-mer analysis (Supplementary Data 3), the 737 accessions were classified into four groups, i.e., hexaploid E. crusgalli and E. colona, and tetraploid E. oryzicola and E. walteri. On average, $97.3 \%$ reads of the E. crus-galli group, $95.4 \%$ of $E$. oryzicola, $84.9 \%$ of E. walteri, and $70.8 \%$ of E. colona were mapped to STB08 (Fig. 2b). For the subgenomes $\mathrm{AH}$ and $\mathrm{BH}$, the average read coverages for E. crus-galli accessions were $93.5 \%$ and $93.2 \%$, respectively, which were higher than those for the accessions of $E$. oryzicola ( $87.6 \%$ and $86.8 \%)$, E. walteri $(73.9 \%$ and $71.4 \%)$, and $E$. colona $(25.0 \%$ and $40.1 \%)$, consistent with the fact that $E$. oryzicola is an ancestral parent of E. crus-galli. For subgenome $\mathrm{CH}$, the average read coverage for E. crus-galli accessions was $95.6 \%$, while it was only $11.9 \%$ and $12.6 \%$ for E. oryzicola and E. walteri accessions, respectively, because of their lack of subgenome $\mathrm{CH}$. However, E. colona accessions had $78.0 \%$ average read coverage on subgenome $\mathrm{CH}$, indicating that subgenome $\mathrm{DH}$ of E. colona is genetically close to subgenome $\mathrm{CH}$ of E. crus-galli (Fig. 1d).

Our global collection was composed of 596 accessions of hexaploid E. crus-galli, 32 of hexaploid E. colona, 85 of tetraploid E. oryzicola, 15 of tetraploid E. walteri, and nine outliers (with abnormal read mapping rates or genome coverage; see details in next section). The accessions from China (90.0\%), Brazil (86.7\%), and Malaysia (94.7\%) were dominantly E. crus-galli, while those from Italy were mainly E. oryzicola (89.3\%). Echinochloa accessions from the United States were diverse, with the highest Simpson's diversity index of 0.676 , compared to 0.182 from China, 0.100 from Malaysia, 0.231 from Brazil, and 0.191 from Italy (Fig. 2a).

We called single nucleotide polymorphisms (SNPs) for subgenome A (9.55 million and 20.2 SNPs/kb), B (6.56 million and 16.4 SNPs/kb), and C (4.60 million and 11.0 SNPs/kb) and used them in population structure and phylogenetic analyses for identifying varieties in each group (Fig. 2c; Supplementary Fig. 20). In E. oryzicola, a clade from Hainan, China (designated E. oryzicola var. hainanensis), showed signs of genetic admixture, despite similar read mapping rates and genome coverage as the other ordinary E. oryzicola accessions (designated E. oryzicola var. oryzicola). The accessions of the E. oryzicola var. hainanensis clade showed distinctive morphological characteristics, including prostrate tillers, small awnless spikelets, and purple panicles, and thus were easily distinguishable from E. oryzicola var. oryzicola accessions, which showed compact plant architectures, large spikelets with long awns, and green panicles (Fig. 2d; Supplementary Figs. 21 and 22; Supplementary Note 1). In E. crus-galli, five varieties (four weedy forms, including var. crus-galli, var. crus-pavonis (Kunth) Hitchc., var. praticola Ohwi, and var. oryzoides (Ard.) Lindm., and one cultivated form, i.e., var. esculenta) and two admixture groups could be determined (Fig. 2c; Supplementary Fig. 20). In the weedy forms, var. crusgalli and var. oryzoides usually have smaller tiller angles as a typical feature of rice mimicry (Fig. 2d; Supplementary Fig. 22). Var. oryzoides has the largest grain size or weight among all $E$. crus-galli varieties, even comparable to that of E. oryzicola var. oryzicola (Fig. 2d; Supplementary Fig. 21). The population structure clustering was supported by a principal component analysis (Supplementary Fig. 23). Although E. crus-galli was dominant in China, Brazil, and Malaysia, at the variety-level, var. crus-galli was predominant in paddy fields of China, while var. crus-pavonis was prevalent in Brazil and admix1 in Malaysia. In China, the northwestern and southern paddy fields were mainly infested by var. oryzoides and var. crus-galli, respectively (Supplementary Data 3).

The demographic history of E. crus-galli was further inferred using fastsimcoal2 based on the unfolded joint site frequency spectrum (SFS) of unlinked SNPs synonymous with known ancestral alleles ${ }^{34}$. Among the five divergence history scenarios, the best-fit scenario was the hybrid origin of E. crus-galli var. cruspavonis with continuous gene flow from var. crus-galli to var. crus-pavonis (Fig. 2e; Model 5 in Supplementary Fig. 24 and Supplementary Table 1) according to the Akaike information criterion (AIC) score. The speciation of E. crus-galli was inferred to have occurred 283 thousand years ago, consistent with the estimation based on $K_{s}$. Var. crus-pavonis originated from the admixture between var. crus-galli $(20.2 \%)$ and common ancestors (79.8\%) of var. praticola, var. oryzoides and var. esculenta 183 thousand years ago, corresponding to the admixed genetic components in population structure when $K=3$ and 4 

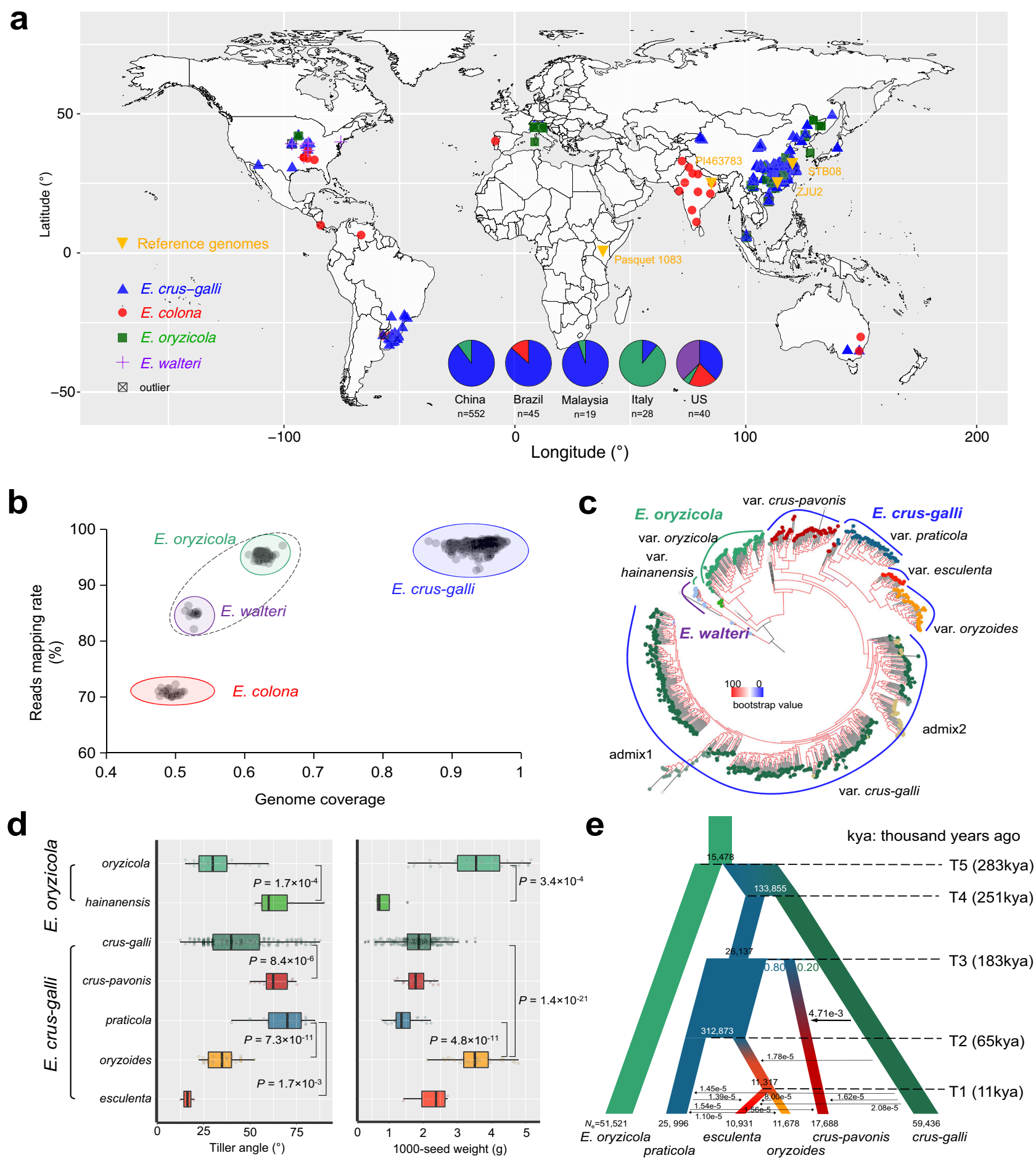

Fig. 2 Population structure and demography of Echinochloa species. a Global distribution of the Echinochloa plants used in this study. The locations of the four Echinochloa accessions with sequenced reference genomes are indicated by yellow downward triangles. The species distributions of the Echinochloa accessions sampled from five countries (China, Brazil, Malaysia, Italy, and United States) are shown in pie charts. b Four Echinochloa species are identified based on mapping rates and genome coverage of reads mapped to the $E$. crus-galli reference assembly (STB08). c The maximum-likelihood phylogeny of $E$. crus-galli and E. oryzicola accessions are inferred using genome-wide non-synonymous single nucleotide polymorphisms (SNPs), with tetraploid $E$. walteri as an outgroup species. d Phenotypic differences (tiller angle and 1000-seed weight) in the varieties of E. oryzicola and E. crus-galli (accession number: $n=38$ oryzicola, $n=6$ hainanensis, $n=321$ crus-galli, $n=15$ crus-pavonis, $n=25$ praticola, $n=38$ oryzoides, $n=4$ esculenta). In the box plots, the horizontal line shows the median value, and the whiskers show the $25 \%$ and $75 \%$ quartile values of tiller angles and seed weights. $P$ values are calculated using the twotailed Wilcoxon test. e Demographic history of $E$. crus-galli varieties. T1 to T5 indicate the estimated time of population divergence. $N_{\mathrm{e}}$, effective population size. Kya, thousand years ago. Source data are provided as a Source data file. 
(Supplementary Fig. 20). The ancestral population of var. oryzoides and var. esculenta diverged from var. praticola 65 thousand years ago, and var. esculenta was domesticated 11 thousand years ago. In addition, frequent gene flow events were detected among varieties, especially after the emergence of agriculture ( $\sim 10$ thousand years ago) (Fig. 2e; Supplementary Fig. 25; Supplementary Data 4). Considering that barnyard grasses are crop companions, especially of rice, these results suggest that the agricultural activities and germplasm exchange via trading may have facilitated their dispersal and gene flow among varieties ${ }^{35}$.

Recent history within the last 10 thousand years was examined based on both folded and unfolded SFS using Stairway Plot $^{36}$ (Supplementary Fig. 26). A sharp bottleneck was observed in var. praticola at $\sim 3000$ years ago. Population sizes were constrained, beginning at 1000 years ago for var. crus-pavonis and 400 years ago for var. crus-galli. After recovering from a bottleneck around 10,000 years ago, the population size of var. oryzoides remained stable in the recent 2000 years. Within the last 100 years, the effective population size of cultivated var. esculenta decreased severely and continuously.

Generally, an asymmetric distribution of nucleotide diversity $\left(\pi_{\mathrm{A}}>\pi_{\mathrm{B}}>\pi_{\mathrm{C}}\right)$ was observed in Echinochloa species (Supplementary Fig. $27 \mathrm{a}, \mathrm{b})$, similar to that in wheat ${ }^{37}$. For example, subgenome $\mathrm{C}$ showed the lowest diversity among the three subgenomes in E. crus-galli varieties, similar to the lowest diversity observed in subgenome $\mathrm{D}$ of wheat. This could be explained by the severe bottleneck in subgenome $\mathrm{C}$ during hexaploidization or increased diversity in subgenomes A and B after the speciation of E. crus-galli. In contrast, the differences between the diversities of subgenome A or B and subgenome $\mathrm{C}$ were larger in var. crus-galli than in the other four varieties, implying that var. crus-galli experienced more dynamic genomic alterations, which increased the nucleotide diversity of subgenomes A and B (Supplementary Fig. 27).

Sympatric gene flow among Echinochloa weeds. Interspecific introgression from ancestral populations is known to have played a primary role in increasing genetic diversity in various genera. For example, the genetic diversity of hexaploid bread wheat was enhanced by introgression from tetraploid emmer and other wheats ${ }^{37,38}$. We constructed an ML phylogenetic tree using SNPs in Echinochloa chloroplast genomes with S. italica as an outgroup (Fig. 3a). Echinochloa colona formed a monophyletic group (clade 0 ) and was located at the root. The remaining Echinochloa taxa were clustered into two clades (clade 1 or clade 2). Echinochloa haploclada, E. walteri, E. oryzicola var. hainanensis, and most E. crus-galli accessions were clustered in clade 2, while E. oryzicola var. oryzicola and some E. crus-galli var. crus-galli accessions were clustered in clade 1, although var. crus-galli in clade 1 and clade 2 was mixed together in the nuclear tree (Figs. $3 a$ and $2 c$ ). It was previously reported that E. oryzicola var. oryzicola was assumed to be a male donor in the polyploidization of hexaploid E. crusgalli $^{16}$. Here, the chloroplast phylogeny indicated that at least two male donors contributed to the origin of E. crus-galli. This is discordant with the hypothesis of a single origin inferred from nuclear genome phylogeny. Chloroplast capture may underlie the polyphyletic plastid phylogeny. Either sexual hybridization or asexual grafting can lead to chloroplast capture or horizontal transfer ${ }^{39}$. While natural grafting is common in woody plants, it has never been reported in grasses ${ }^{40}$, whereas interspecific hybridization has been frequently reported in grasses.

To examine for potential introgression (gene flow) from $E$. oryzicola var. oryzicola to E. crus-galli var. crus-galli, four topologies were set in ABBA-BABA statistics (Fig. 3b; Supplementary Fig. 28).
Compared to the null hypothesis (T1), significantly higher $f_{\mathrm{d}}$ values (the proportion of introgression) were observed across the nuclear genome in topologies T2, T3, and T4 (Supplementary Fig. 28). The proportions of genome introgression estimated by PGI (an index to reflect genome-wide introgression level) ${ }^{37}$ were higher in topologies T2, T3, and T4 compared to the null topology. The relationship between windowed $f_{\mathrm{d}}$ and nucleotide diversity $\pi$ confirmed that introgression has elevated the overall genetic diversity of subgenomes $\mathrm{A}$ and $\mathrm{B}$ in var. crus-galli, with $\pi_{\mathrm{A}}=1.61 \times 10^{-3}$ and $\pi_{\mathrm{B}}=1.36 \times 10^{-3}$ when $f_{\mathrm{d}}>0.02$, compared to $\pi_{\mathrm{A}}=1.14 \times 10^{-3}$ and $\pi_{\mathrm{B}}=1.07 \times 10^{-3}$ when $f_{\mathrm{d}}<0.02$. However, a proportion of windows with $f_{\mathrm{d}}$ values close to 1 showed extremely low $\pi$ values, suggesting that the fixation of introgressed segments would not increase diversity; instead, the median magnitude of introgression would increase to the maximum extent, similar to the pattern in wheat (Supplementary Fig. 29).

Genes related to stress response were enriched in introgressed segments with high frequency $\left(f_{\mathrm{d}}>0.5\right)$ (Supplementary Fig. 30). For example, a large region from $7.0 \mathrm{Mb}$ to $9.0 \mathrm{Mb}$ on chromosome AH05 had an extreme $f_{\mathrm{d}}$ value close to 1 . This region has a total of 34 genes, including seven biotic and 12 abiotic stress response genes (Fig. 3b).

Echinochloa oryzicola var. oryzicola and E. crus-galli var. crusgalli have overlapping distributions and ecological niches (Supplementary Data 3; Supplementary Note 1). Three E. crusgalli accessions (QX6, JX9, and JX111) from China were scattered within E. oryzicola var. oryzicola in the chloroplast tree, reflecting the recurrent hybridization events between E. oryzicola var. oryzicola and var. crus-galli (Fig. 3a). To examine whether they were recent hybrids, re-sequenced reads were mapped to a decaploid pseudo-genome created by combing the STB08 and ZJU2 reference assemblies. For E. oryzicola var. oryzicola and E. crus-galli var. crus-galli accessions without introgression, the mapping depths in the five subgenomes were biased (Fig. 3c), while for accessions JX111 and QX6, the subgenome mapping depths were symmetric, clearly demonstrating the recent hybridization between E. oryzicola and E. crus-galli. However, for JX9, the biased mapping depths revealed that small introgression footprints were retained in nuclear genomes as a result of back-crossing with E. crus-galli, after an ancient interspecies hybridization event, which led to chloroplast genome substitution. These results were consistent with admixed genomic compositions revealed by population structure analysis (Supplementary Fig. 31). Based on this method, we found that six of the nine outliers (identified by the abnormal rate of read mapping and genome coverage against E. crus-galli reference genome STB08) in our collection were recent hybrids with multiple subgenomes from more than one species (Supplementary Data 3; Supplementary Fig. 31).

Local adaptation to natural environments. Distribution of $E$. crus-galli varieties showed high differentiation with respect to habitat, with var. crus-galli and var. crus-pavonis mainly found in low altitudes, while var. praticola and var. oryzoides typically occurring in relatively high altitudes (Supplementary Fig. 32). A difference in latitudinal distribution was also observed in $E$. oryzicola, with accessions from higher latitudes (Italy, Northeast China, Korea, and Japan) forming a clade (hereafter referred to as group HL) separated from those occurring in lower latitudes (group LL) (Southern China and Malaysia) (Supplementary Figs. 32 and 33). We calculated the genomic differentiation $\left(F_{\mathrm{st}}\right)$ between var. crus-galli and var. praticola in E. crus-galli and between group LL and HL in E. oryzicola to trace the footprints of local adaptation to natural environments and to explore the potential convergent evolution of the two Echinochloa species. 
a
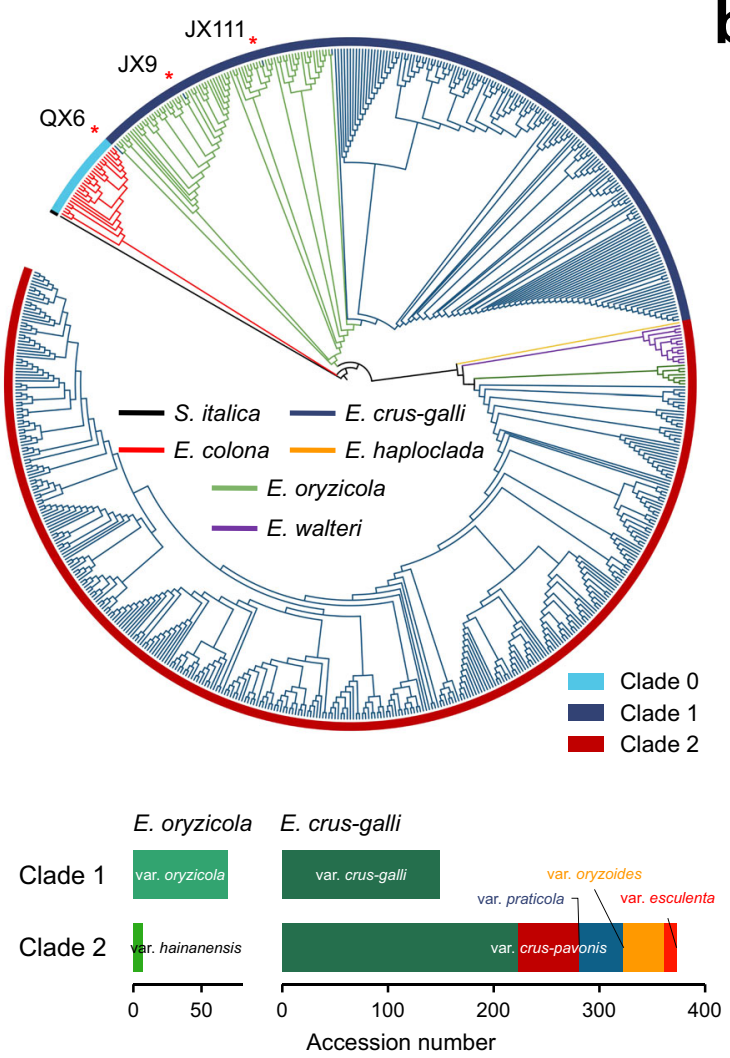

C

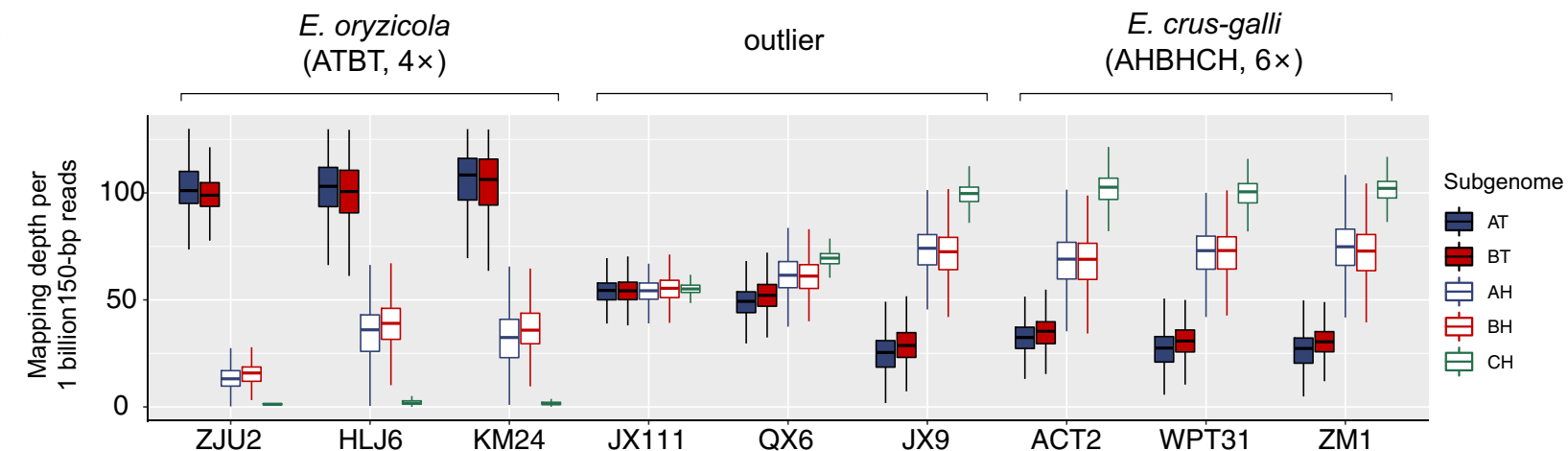

T1: (E. walteri, (E. o. var. oryzicola, (E. c. var. praticola, E. c. var. oryzoides)))
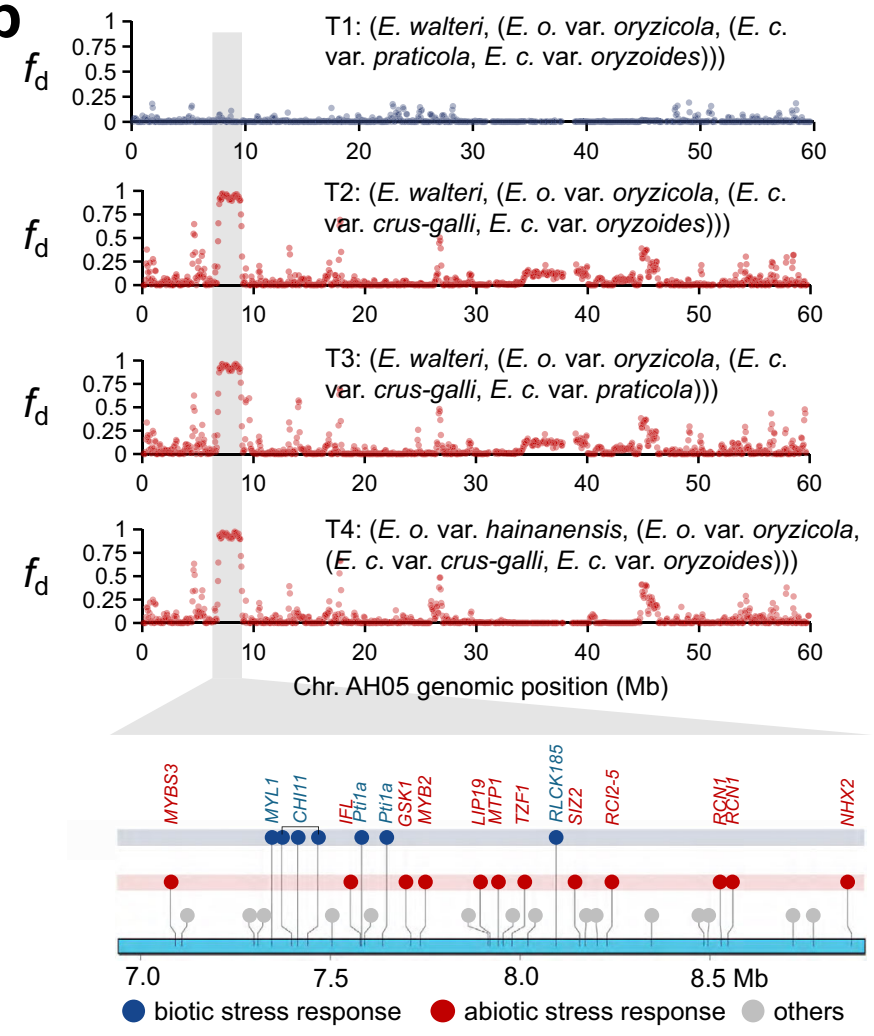

Fig. 3 Sympatric gene flow among Echinochloa species. a A maximum-likelihood phylogeny based on single nucleotide polymorphisms (SNPs) in the
chloroplast genomes of Echinochloa accessions $(n=607)$. Three non-E. oryzicola accessions (QX6, JX111, and JX9) clustered within E. oryzicola var. oryzicola (clade 1) are indicated by red asterisks. Histogram charts below the phylogeny tree show the species and variety compositions in clade 1 and clade 2. b Proportion of introgression $f_{d}$ distributions of ABBA-BABA test on chromosome AH05 in topology T1-T4 (see details in Supplementary Fig. 28). Stressresponsive genes with sequences ranging from $7 \mathrm{Mb}$ to $9 \mathrm{Mb}$ on chromosome AH05 are shown in the lower panel. c Mapping depths when reads from three $E$. oryzicola accessions, three E. crus-galli accessions, and three outliers are mapped against the pseudo-decaploid reference genome (STB08 + ZJU2). In the box plots, the horizontal line shows the median value, and the whiskers show the $25 \%$ and $75 \%$ quartile values of mapping depths of $1-\mathrm{Mb}$ sliding windows in five subgenomes (window number: $n=506 \mathrm{AT}, n=421 \mathrm{BT}, n=478 \mathrm{AH}, n=405 \mathrm{BH}, n=421 \mathrm{CH}$ ).

Flowering time (FT) is a crucial fitness trait in local adaptation. In E. crus-galli, var. crus-galli flowered significantly later than var. praticola and var. oryzoides $\left(P=5.7 \times 10^{-11}\right.$ and $P=2.1 \times 10^{-161}$, respectively, Student's $t$-test), and in E. oryzicola, the accessions from group HL flowered earlier than those from group LL $\left(P=2.6 \times 10^{-6}\right.$, Student's $t$-test $)$, as found in our common-garden experiments (Supplementary Fig. 32). A total of 13 genes (7 in subgenome A, 5 in B, and 1 in C) regulating FT were found to be highly differentiated (top $1 \%$ in $F_{\text {st }}$ windowed values) in E. crusgalli, including $F T, H d 1$, Ehd1, and DTH2 (Fig. 4a; Supplementary Fig. 34; Supplementary Data 5). Among these genes, Hd1
(AH06.1150), located on chromosome 6 of subgenome A, was also identified during E. oryzicola differentiation (Fig. 4a; Supplementary Data 6). A test of selection (Tajima's D) and nucleotide diversity $(\pi)$ analyses showed that a $9-\mathrm{Mb}$ genomic region extending from 13 to $22 \mathrm{Mb}$ on chromosome AH06, harboring $H d 1$, was under strong positive natural selection in var. crus-galli (Fig. 4b; Supplementary Fig. 35). A genome-wide association study (GWAS) for the FT of E. crus-galli also identified Hd1 (Fig. 4b; Supplementary Data 7). Selection on Hd1 was similarly detected in var. crus-pavonis and group LL that shared the late-flowering trait with var. crus-galli, implying that 

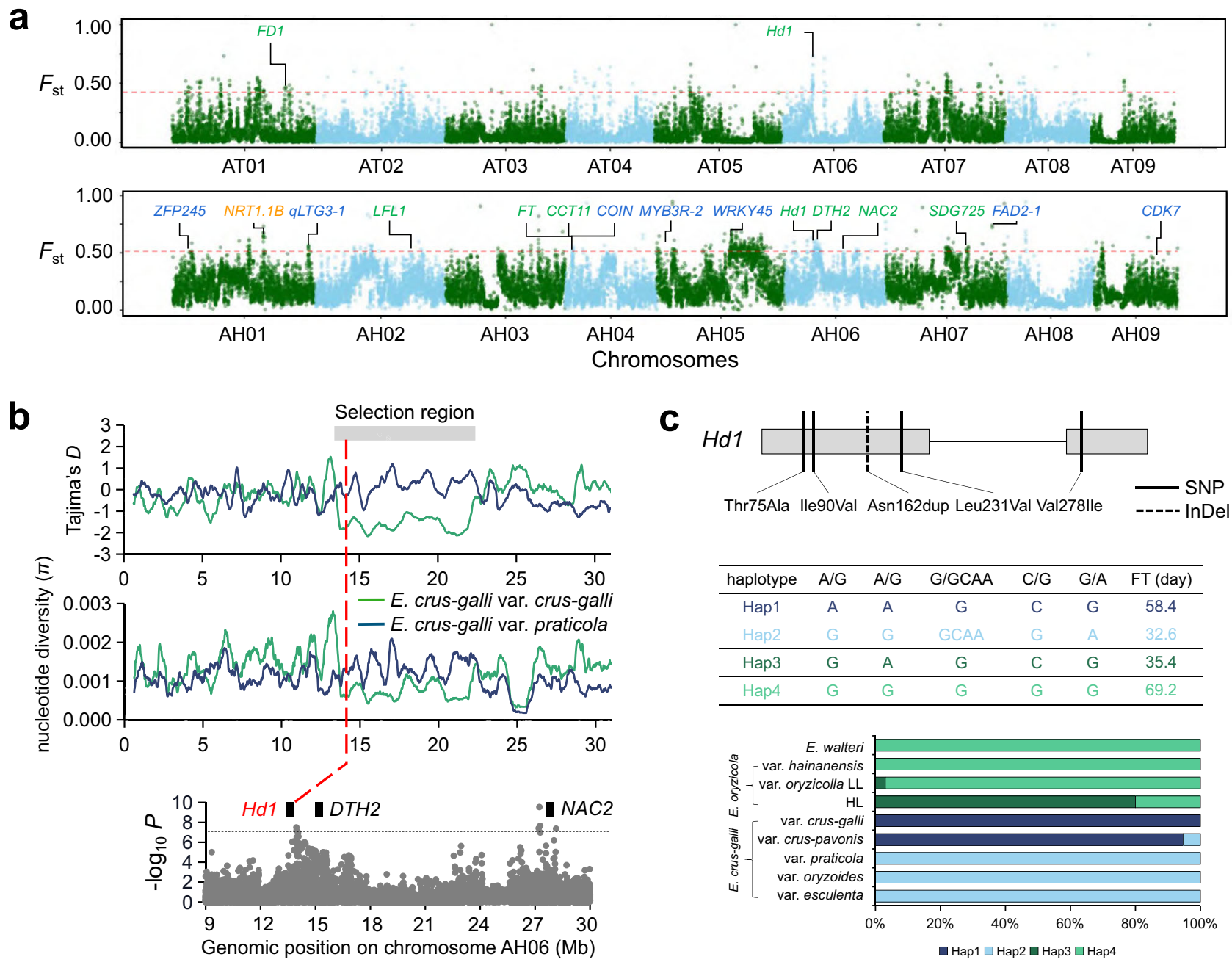

\begin{tabular}{ccccccc}
\hline haplotype & A/G & A/G & G/GCAA & C/G & G/A & FT (day) \\
\hline Hap1 & A & A & G & C & G & 58.4 \\
\hline Hap2 & G & G & GCAA & G & A & 32.6 \\
\hline Hap3 & G & A & G & C & G & 35.4 \\
\hline Hap4 & G & G & G & G & G & 69.2 \\
\hline
\end{tabular}

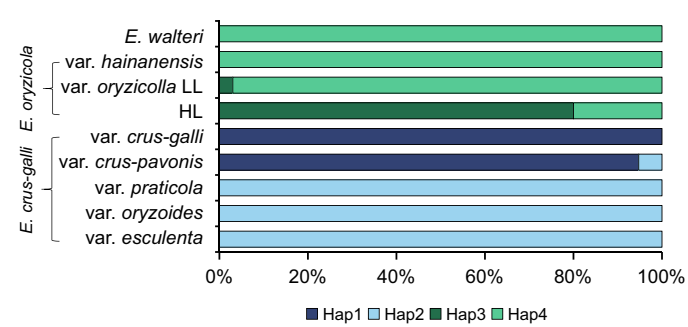

Fig. 4 Local adaptation of Echinochloa accessions. a Genomic differentiation $\left(F_{\mathrm{st}}\right)$ between high-latitude $(\mathrm{HL})$ and low-latitude (LL) E. oryzicola var. oryzicola populations (upper panel), and between E. crus-galli var. praticola and var. crus-galli (lower panel). Genes related to flowering (green) and cold tolerance (blue) in the top $1 \% F_{\mathrm{st}}$ windows (red horizontal dashed lines) in E. oryzicola and E. crus-galli subgenomes A are shown, respectively. b Selection features (Tajima's $D$ and nucleotide diversity $\pi$ ) of $H d 1$ and genome-wide association study on flowering time in E. crus-galli. The red dashed line indicates the genomic position of $\mathrm{Hd} 1$ on chromosome AH06. c Analysis of $\mathrm{Hd} 1$ haplotypes in E. oryzicola and E. crus-galli. Four haplotypes composed by four SNPs and one InDel show differences in flowering time. Source data are provided as a Source data file.

Hd1 plays essential roles in regulating FT in Echinochloa. Given that E. crus-galli and E. oryzicola are polyploids, the profiling of $H d 1$ genes in other subgenomes was scanned. No obvious signal of differentiation (indicated by $F_{\text {st }}$ ) and selection (indicated by Tajima's $D$ and $\pi$ ) was found for the $H d 1$ genes (BH06.1184 and $\mathrm{CH} 06.1302)$ in subgenomes $\mathrm{B}$ and $\mathrm{C}$, suggesting preferential evolution among subgenomes of Echinochloa in environmental adaptation (Supplementary Fig. 36).

We identified four Hd1 haplotypes, composed of five functionally important and divergent non-synonymous polymorphisms (four SNPs leading to amino acid substitution and one 3-bp insertion leading to an Asn duplication) (Fig. 4c). Accessions with Hap1 and Hap4 showed significantly later flowering (58.4 and 69.2 days, respectively) relative to those with Hap2 and Hap3 (32.6 and 35.4 days, respectively) $\left(P=3.6 \times 10^{-32}\right.$ between Hap1 and Hap2, $P=4.1 \times 10^{-9}$ between Hap3 and Hap4, Student's $t$-test) (Fig. 4c). Hap3 and Hap4 were only found in tetraploid E. walteri and E. oryzicola, and Hap1 and Hap2 only in E. crus-galli. These observations imply that $H d 1$ functioned in parallel in the natural adaptation of E. oryzicola and E. crus-galli.
Furthermore, it is worth noting that var. praticola is predominantly found in dry lands, compared to var. crus-galli, var. oryzicola, and var. crus-pavonis, which occur in the lowlands, especially paddy fields (Supplementary Note 1). Besides differentiation at the level of macroscopic climate adaptation between var. praticola and var. crus-galli, genes underlying habitat preference may also be divergent. Gene ontology (GO) terms related to stress response (GO:0006979, response to oxidative stress, $P=0.042$; GO:0009415, response to water, $P=0.0049)$ and nitrogen utilization (GO:0008519, ammonium trans-membrane transporter activity, $P=0.022$; GO:0015696, ammonium transport, $P=0.022$ ) were enriched in highly differentiated genomic regions. A homolog of NRT1.1B (AH01.2967) located on chromosome AH01 showed high differentiation potential $\left(F_{\mathrm{st}}=0.63\right)$ (Fig. $\left.4 \mathrm{a}\right)$. NRT1.1B has been reported as a key transporter in regulating differential nitrogen utilization between japonica and indica rice types ${ }^{41}$. The haplotype of NRT1.1B diverged between var. praticola and other varieties of $E$. crus-galli, with four amino acid substitutions, suggesting that var. praticola may have a differential nitrogen utilization ability compared to other varieties, allowing it to grow well in lownitrogen-content uplands (Supplementary Fig. 37). 

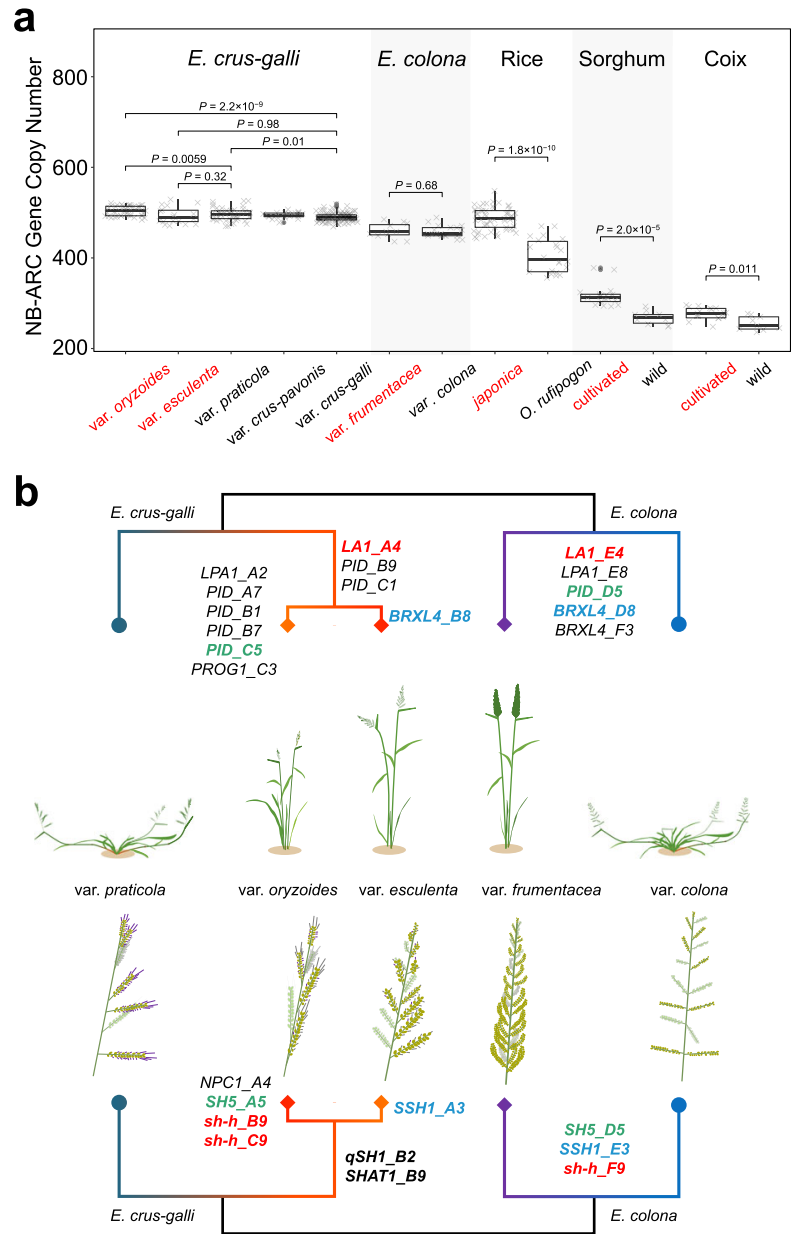

Fig. 5 Domestication of barnyard millets. a Alteration of the NB-ARC gene family size during domestication for five crops (two barnyard millets, rice, sorghum and coix). E. crus-galli: $n=43$ oryzoides, $n=18$ esculenta, $n=48$ praticola, $n=22$ crus-pavonis, $n=115$ crus-galli; E. colona: $n=11$ frumentacea, $n=19$ colona; rice: $n=55$ japonica, $n=22$ wild; sorghum: $n=18$ cultivated, $n=10$ wild; Coix: $n=18$ cultivated, $n=9$ wild. In the box plots, the horizontal line shows the median value, and the whiskers show the $25 \%$ and $75 \%$ quartile values of NB-ARC gene copy numbers in five species. $P$ values are calculated using the two-tailed Wilcoxon test. $\mathbf{b}$ Genomic divergence of key genes regulating plant architecture and grain shattering between barnyard millets and grasses. Chromosome IDs are indicated after the gene names. For example, 'LA1_A4' represents the gene $L A 1$ on chromosome AHO4. Source data are provided as a Source data file.

Facilitation of herbicide resistance gene discovery in barnyard grass populations. ALS inhibitor herbicides are among the most widely used worldwide, and consequently resistance evolution (both target-site- and non-target-site-based) to these herbicides exceeds that of any other site-of-action herbicide ${ }^{11,13,42,43}$. Genome resequencing of the barnyard grass samples from the paddy fields of Brazil, Italy, and China revealed three known ALS resistance mutations (Ala122Thr, Trp574Leu, and Ser653Asn) and a Gly654Cys substitution that has not been reported previously in plants (Supplementary Data 8). Each of these ALS resistance mutations was associated with the resistance phenotype and occurred preferably in subgenome A (coincided with enriched TE) (Supplementary Data 8 and 9). In addition, most ALS resistance mutations were found to occur in a homozygous state (especially for the mutations at amino acid positions 122, 653, and 654); this apparent selection for homozygosity could potentially be a mechanism to overcome dilution effects by the wild-type (susceptibility) homeo-alleles across the genome.

Quinclorac is a highly selective auxin herbicide for barnyard grass control in rice fields. Resistance to quinclorac in barnyard grasses renders this herbicide ineffective in rice-producing countries, including China, Brazil, and USA. Despite much effort, the molecular basis of quinclorac resistance remains elusive $^{44}$. Recently, a point mutation in the conserved degron region of auxin receptor AUX/IAA16 and a 27-bp deletion in the degron tail region of AUX/IAA2 have been demonstrated to endow resistance to other auxin herbicides (e.g., 2,4-D) in weedy plant species ${ }^{45,46}$. In light of these discoveries and taking advantage of our genome data, a total of 60 AUX/IAA genes were identified in the E. crus-galli genome (domain prediction as Aux/IAA gene family with the degron region 'VGWPPV') and compared for SNPs and InDels (small insertions and deletions) between the Brazilian samples of resistant and susceptible phenotypes. An Arg86Gln mutation in the conserved degron tail region of Echinochloa AUX/IAA12 was identified in one sample (SA88) with the resistance phenotype (Supplementary Figs. 38 and 39), and it is a potential candidate target-site gene for quinclorac resistance, pending for further confirmation at the population, genetic, and functional levels.

Apart from target-site resistance, non-target-site resistance (NTSR) also endows herbicide resistance ${ }^{47,48}$. Indeed, some Brazilian and Italian samples with the multiple resistance phenotypes had no ALS and ACCase mutations, indicating NTSR to herbicides, usually relating to reduced herbicide uptake/ translocation and enhanced herbicide metabolism, involving super families of ABC transporters, cytochrome P450 (CYP450), and glutathione S-transferase (GST) ${ }^{47,48}$. Convergent evolution of gene copy number variation (CNV) has been shown in target-site resistance to glyphosate in many weedy species ${ }^{48,49}$. To investigate whether NTSR to ALS inhibitors is related to CNV, we estimated the copy numbers of CYP450, GST, and ABC transporter genes in the Brazilian E. crus-galli samples lacking ALS target-site mutations and observed no amplification of CYP450 and GST genes but slight expansion of ABC transporter genes $(P<0.05$, Student's $t$-test) (Supplementary Data 9$)$, indicating the existence of other NTSR mechanisms that are not related to CNV. A glyphosate-detoxifying aldo-keto reducatse (AKR) and glyphosate-exporting ABCC8 transporter have been cloned and characterized from barnyard grass in our previous studies, and a higher expression of these genes was found to confer glyphosate resistance ${ }^{50,51}$. It is interesting to determine whether the CNV of these genes is involved and the underlying genomic mechanism. Our current study annotated 867 CYP450s, 227 GSTs, 113 AKRs, and $361 \mathrm{ABC}$ transporters in the E. crus-galli genome, and 694, 226,111 , and 336 of each category in the E. colona genome. These are rich resources vital to gene discovery in weedy plants for stress adaptation in general and for herbicide NTSR in particular.

Domestication of barnyard millets. As noted above, domestication events occurred in at least two hexaploid Echinochloa species, E. crus-galli and E. colona. From the phylogenetic analysis results (Fig. 2c; Supplementary Fig. 40), we inferred that E. crusgalli var. esculenta and E. colona var. frumentacea, mainly cultivated in East Asia and India, respectively ${ }^{19,52}$, were domesticated from E. crus-galli var. praticola and E. colona var. colona, respectively. Interestingly, despite being a paddy weed, E. crusgalli var. oryzoides has domestication-like traits, for instance, the largest grain size among all E. crus-galli varieties and wheat-like plant architecture (Fig. 5b; Supplementary Note 1).

Like other crops, nucleotide diversity in barnyard millets decreased during domestication. For example, in E. crus-galli, the 
nucleotide diversities of var. esculenta $\left(\pi=0.865 \times 10^{-3}\right)$ and var. oryzoides $\left(\pi=0.773 \times 10^{-3}\right)$ were significantly lower than the nucleotide diversity of var. praticola $\left(\pi=1.021 \times 10^{-3}\right)$ (Supplementary Fig. 27). The genetic distances estimated for linkage disequilibrium (LD) decay (indicated by $r^{2}$ ) to half of the highest value were obviously longer across whole genomes in the two $E$. crus-galli domesticates (E. crus-galli var. esculenta, $r^{2}=0.32$, $28 \mathrm{~kb}$; var. oryzoides, $r^{2}=0.29,35 \mathrm{~kb}$ ) than in the weed populations (E. crus-galli var. praticola, $r^{2}=0.26,0.6 \mathrm{~kb}$ ). However, the LD magnitudes were similar between the cultivated and wild/weed forms in E. colona ( $91 \mathrm{~kb}$ for E. colona var. frumentacea when LD decayed to $r^{2}=0.39 ; 89 \mathrm{~kb}$ for $E$. colona var. colona when LD decayed to $r^{2}=0.37$ ) (Supplementary Fig. 41), implying a domestication impact of lower magnitude on the genome of E. colona.

Artificial selection during domestication and improvement has increased the diversity and size of the NB-ARC family to improve immune responses in rice ${ }^{27}$, wheat ${ }^{10}$, and foxtail millet (336 in foxtail grass versus 368 in foxtail millet) (Fig. 1d). We developed a method to infer the domestication magnitude of barnyard millets by comparing the NB-ARC family size in the accessions of cultivated and wild species based on the abundance of reads generated by high-depth resequencing (Supplementary Note 6; Supplementary Data 10). We first tested the method in rice, sorghum, and the orphan crop Job's tears (Coix lacryma-jobi) and achieved a result consistent with that generated based on comparisons using reference genomes in all three cases, i.e., the NB-ARC family size was significantly larger in cultivated species than in their corresponding wild progenitors (Fig. 5a). In these cases, the size of the NB-ARC family appears to increase with the level of domestication (i.e., japonica rice $>$ sorghum $>$ Job's tears). However, in barnyard millets, no increase was observed in the NB-ARC family size in either the cultivated E. colona var. frumentacea or the cultivated E. crus-galli var. esculenta, implying that their domestication intensity was low (Fig. $5 \mathrm{a} ; P=0.011$ for coix, $P=2.0 \times 10^{-5}$ for sorghum, and $P=1.8 \times 10^{-10}$ for rice, Wilcoxon test). Detection of significantly greater number of NBARC genes in E. crus-galli var. oryzoides than in other Echinochloa varieties supports the speculation of E. crus-galli var. oryzoides being an ancient crop ( $P=0.0059$, Wilcoxon test).

We scanned the allele frequency spectrum of non-synonymous variation in crucial genes underling key domestication traits (e.g., plant architecture, shattering loss, and grain size $)^{53}$. In the gravity response pathway associated with regulation of tiller angles, eight non-synonymous variations in four homologs (LA1, BRXL4, and two PID genes) were found to be highly divergent between $E$. crus-galli var. esculenta and E. crus-galli var. praticola, and 16 non-synonymous substitutions in nine homologs (LA1, LPA1, PROG1, and six PID genes) were found to be highly divergent between E. crus-galli var. oryzoides and E. crus-galli var. praticola (Fig. 5b; Supplementary Data 11). In E. colona, seven nonsynonymous variations in five homologs (LA1, LPA1, PID, and two BRXL4 genes) were divergent between the cultivated and weedy E. colona. We noticed that LA1 on chromosome 4 was highly divergent in three domesticates, and the LA1 homologs on chromosome AH04 and EH04 were in perfect syntenic relationship, suggesting that parallel selection targeted these genes during the domestication of E. crus-galli and E. colona (Supplementary Fig. 42). LA1_A4 (LA1 on chromosome AH04) was divergent from E. crus-galli var. praticola in the common ancestor of var. esculenta and var. oryzoides by four amino acid replacements (Lys107Glu, Leu211Phe, Arg290Lys, and Lys340Asn), while the divergence in LA1_E4 of E. colona was due to another amino acid change (Arg229Cys) (Supplementary Data 11). The $F_{\text {st }}$ around the six $L A 1$ copies on chromosome 4 in the two species indicated that not only $L A 1-A 4$, but also $L A 1 \_B 4$ and $L A 1 \_C 4$ showed high differentiation in the two E. crus-galli domesticates, in contrast to high differentiation on only chromosome EH04 in E. colona (Supplementary Fig. 42; Supplementary Data 12-14). Two syntenic PID copies on chromosome 5 were both selected in $E$. crus-galli var. oryzoides and E. colona var. frumentacea, and two syntenic BRXL4 copies were shared by E. crus-galli var. esculenta and E. colona var. frumentacea. Both revealed parallel selection on syntenic homologs in independent domestication events (Fig. 5b; Supplementary Fig. 43; Supplementary Data 11). For shatteringrelated homologous genes, non-synonymous variants were found to have experienced selection in $s h-h, S H 5, S S H 1, S H A T 1, q S H 1$, and NPC1 genes in three barnyard millets (Fig. 5b; Supplementary Fig. 43; Supplementary Data 11). In grain enlargementrelated homologous genes, a total of 81 non-synonymous variants were found to be differentiated between barnyard millets and grasses in key genes controlling grain size, including GS5, TGW6, and $q G W 8$ (Supplementary Data 11).

\section{Discussion}

Polyploidy plays an important role in plant genome evolution $^{54,55}$. To date, the genomes of five hexaploids have been sequenced and bread wheat is the sole hexaploid member to have been assembled at the chromosome level ${ }^{56,57}$. The genomes of two allohexaploid Echinochloa species (E. crus-galli and E. colona) used in this study are among the most compact hexaploid genomes $(<1.5 \mathrm{~Gb})$ currently known in plants (https:// cvalues.science.kew.org/), with less than one-tenth of the genome size of bread wheat $(\sim 17 \mathrm{~Gb})$. They, therefore, are an ideal model system for studying plant polyploidization and the impact of the events, such as complex interactions among subgenomes and genomic dynamics following polyploid speciation. Despite the small genome size, generating an accurate chromosome-level assembly of allohexaploid Echinochloa is still a challenge, due to high level of sequence similarity between subgenomes and the presence of homeologous exchanges. Here we built genome assemblies of Echinochloa, although we believe that the assemblies could be further improved by integrating information from other approaches, such as genetic maps and optical maps.

Accurately distinguishing Echinochloa species is the first step for basic and applied research of the species, including precision weed control, and is still a challenge due to the morphological similarity among the diverse Echinochloa species ${ }^{16}$. We could accurately distinguish Echinochloa species and varieties by integrating morphological characteristics with multiple pieces of genomic evidence, including genome size, read mapping rate, genome coverage, phylogeny, and population structure (Fig. 2; Supplementary Note 1). As a result, we found not only diverse compositions of barnyard grasses in different rice-growing areas, but a new Echinochloa clade (designated E. oryzicola var. hainanensis) (Fig. 2d; Supplementary Note 1). Moreover, we speculate E. crus-galli var. oryzoides being an ancient crop that had once undergone domestication and was subsequently abandoned by farmers based on domestication-like morphological and genomic signatures, such as larger grain size, non-shattering spikelets, lower $\pi$ value, and longer LD decay, as compared to var. praticola (Supplementary Figs. 27 and 41; Supplementary Note 1). This is supported by the evidence that E. macrocarpa, a race of $E$. crus-galli var. oryzoides, has been reported to be harvested as a crop in the Caucasus ${ }^{58}$.

Reticulate evolution occurs commonly in plant speciation, especially in polyploid species complexes ${ }^{59}$. Our whole-genome data analyses imply radiative evolution at the first divergence in Echinochloa and we considered it as an ILS event, based on short interval length and low bootstrap value from species phylogenies, balanced phylogeny proportion in triplet $(\mathrm{AH}, \mathrm{FH}$, and Ehap), 
QuIBL test, and the unbiased distribution of three topologies of triplet (AH, FH, and Ehap) (Supplementary Figs. 14, 16, and 18; Supplementary Data 1). Demographic history of Echinochloa populations is consistent with farming practices (Supplementary Fig. 26). With ancient farming practices, weeds such as var. praticola (a sharp bottleneck at approximately 3000 years ago) with a prostrate plant architecture (a typical trait non-mimic to crops) were more likely to be distinguished and removed by hand weeding. Owing to the popularity of rice transplanting, instead of direct seeding, in China hundreds of years ago, a higher selection pressure was likely applied to barnyard grasses, plausibly resulting in var. crus-galli population contraction. A gap existed in seeding growth between rice and barnyard grass, although var. crus-galli showed a rice-mimic morphology at the seedling stage. The effective population size of cultivated var. esculenta decreased severely and continuously within the last 100 years, likely a result of the recent abandonment of barnyard millet cultivation thanks to improvement of rice planting technologies and management practices that ensured sufficient food supplies and resulted in the replacement of barnyard millets by cold-tolerant rice varieties $^{19,52}$.

Fundamental understanding of herbicide resistance-conferring genes and resistance evolution are important for the development of strategies to delay, mitigate, and manage herbicide resistance. Compared to diploids, resistance gene discovery is more challenging in polyploid weedy species, such as barnyard grasses, because of the presence of multiple homeo-alleles across subgenomes ${ }^{60}$. In this study, the genomic data of global barnyard grasses provide an opportunity to examine variations related to herbicide TSR and rich resources vital to gene discovery for NTSR. Due to exceptionally severe selective pressures, convergent adaptation across species and repeated evolution, herbicide resistance provides an outstanding opportunity to investigate key questions about the genetics of adaptation, in particular the relative importance of adaptation from new mutations, standing genetic variation, or gene flow, with an example of the wellresearched herbicide resistance evolution in Amaranthus tuberculatus ${ }^{61,62}$. The abundant genomic resources of Echinochloa weeds produced in this study would accelerate the research on herbicide resistance evolution.

As typical orphan crops, barnyard millets show excellent resistance to abiotic stresses ${ }^{7}$. Genomic feature analyses revealed that barnyard millets were domesticated only to a low magnitude, in contrast to major crops, such as rice. This kind of 'light' domestication or a wild-/weed-like genome might be critical for orphan crops to adapt to extreme local environments. In the case of barnyard millets, they may have competitive advantages under unfavorable conditions if they have similar genomic features with barnyard grasses. The recently proposed de novo domestication concept of maintaining genetic diversity and environmental adaptability while improving yield potential of wild species, is in line with the genomic feature of orphan crops ${ }^{63}$. Another reasonable explanation is that orphan crops may be non-fully domesticated.

In summary, the genomes of the Echinochloa polyploids produced in this study provide reference genomes for barnyard grasses and millets. These reference genomes together with the profiling of global Echinochloa accessions detailed here will accelerate research in weed evolutionary biology, interaction between crop and weed, and development of novel weed control strategies under the future climate change scenarios.

\section{Methods}

Genome sequencing and de novo assembly. Total DNA for genome sequencing was extracted from the young leaves of a cultivated E. colona accession PI463783 using the cetyltrimethylammonium bromide (CTAB) method. DNA libraries for single-molecule real-time (SMRT) PacBio genome sequencing were constructed following the standard protocols of the Pacific Biosciences Company and sequenced on the PacBio Sequel II platform using the circular consensus sequencing (CCS) approach. Raw reads were corrected using an error correction module Hifiasm (v0.12-r304) ${ }^{64}$ was used for the initial assembly using HiFi reads with parameters '-a 4 -n 3 -x 0.8 -y 0.2.' After mapping the long subreads to the initial assembly with minimap2 (v2.15-r905) ${ }^{65}$, racon (v1.4.3) ${ }^{66}$ was used for correction for three rounds under default parameters. Gmap (v2014-05-15) ${ }^{67}$ was used to annotate the draft assembly using the coding sequences (CDS) of diploid E. haploclada, and the synteny between them was conducted according to the physical orders along chromosomes. The contig-level draft assemblies of E. oryzicola and E. crus-galli were generated by our previous study using PacBio continuous long-read (CLR) sequencing ${ }^{10}$.

Genome scaffolding. Following the standard protocol, we constructed $\mathrm{Hi}-\mathrm{C}$ libraries of E. colona using the fresh young leaves with the digestion of a 4-cutter restriction enzyme MboI. Hi-C sequencing libraries were sequenced on Illumina HiSeq 2500 platform with $2 \times 150$-bp reads. Valid ligation products were used in pseudo-molecule construction with DipHiC pipeline (see details at Supplementary Note 2). In the first module "subgenome distinguishing", we integrated three clustering algorithms to distinguish subgenomes. First, 100-mers split from diploid E. haploclada assembly were mapped to E. colona draft assembly, and the depth of each contig was calculated. According to the biased depths, contigs were clustered into cluster1, cluster2, cluster3, and un-clustered. Second, inter-contig synteny was investigated to construct the contig allelism, which was used to correct previous depth-clustering and re-cluster contigs. Third, by mapping pairs of $\mathrm{Hi}-\mathrm{C}$ reads to draft assembly using Burrows-Wheeler Aligner (BWA; version 0.7.15-r1140) ${ }^{68}$ and filtering, the linkages or interactions were computed among contigs and used for correcting the clustering in the previous step. In the second module 'chromosome building', directional interactions and pre-assignment of chromosome groups were utilized to cluster contigs on each chromosome. Finally, module optimize implemented in $\mathrm{AllHiC}^{69}$ was used to order and orientate contigs within each chromosome.

The procedures of Hi-C library construction and $\mathrm{HiC}$-assisted scaffolding for $E$ oryzicola genome were similar to E. colona (Supplementary Note 3 ). The pipeline of subgenome distinguishing in E. crus-galli was slightly different because of the availability of assemblies of two parental genomes (E. haploclada and E. oryzicola) (Supplementary Note 4). Besides, considering its assembly was based on CLR sequencing with a relative low contig N50, we first corrected the errors in contig assembly by integrating the genetic relationship with parental three subgenome and intra-contig interactions inferred from the Hi-C library and a 20-kb mate-pair library, to rule out the noise of contig mis-assembly. The genomes of E. haploclada and E. oryzicola were both used to distinguish subgenomes in E. crus-galli, and the contig order and orientation were the same as those used previously

(Supplementary Note 4). The genome-wide Hi-C all-to-all interaction heatmaps were generated by AllHiC.

Genome quality assessment. To evaluate the completeness of $E$. colona assembly, BUSCO (v4.1.2) ( $^{70}$ and LTR assembly index (LAI) using LTR retrotransposons ${ }^{71}$ were assessed. The Illumina short reads and RNA-Seq data were used to evaluate the assembly completeness and accuracy by Bowtie $2(\mathrm{v} 2.3 .5 .1)^{72}$. To evaluate the accuracy with which the subgenomes were distinguished, we identified subgenomespecific $K$-mers, which could be used to differentiate homoeologous chromosomes $K$-mers $(K=13)$ were generated using jellyfish (v2.3.0) and the frequency of each $K$-mer was counted for each chromosome ${ }^{73}$. Only the $K$-mers that were enriched in one subgenome ( $K$-mer frequency is $>10$ times that of those in other two subgenomes) were regarded as informative $K$-mers to distinguish subgenomes. The chromosomes and informative $K$-mers were grouped using hierarchical clustering. The assemblies of centromeric and telomeric regions indicate the reliability of the assembly. Tandem repeat finder (v4.09) ${ }^{74}$ was used to identify tandem repeats along the chromosomes. To identify the centromeric regions, the largest repeat arrays composed of 155-bp units were identified and clustered. The telomeric repeats were identified by searching chromosome ends for short high-copy number repeats with the monomer 'AAACCCT'. The accuracy of subgenome distinguishing in E. oryzicola and E. crus-galli was validated by subgenome-specific $K$ mers and transposons. The centromeric regions were also identified on each chromosome of the E. oryzicola and E. crus-galli assemblies.

Genome annotation. The repeat elements were annotated using approaches described previously 9 . A hybrid strategy integrating ab initio predictions by Fgenesh ${ }^{75}$ and AUGUSTUS (v3.2.2) ${ }^{76}$, homoeologous gene evidence, and transcriptomic supports (see RNA-Seq data details in Supplementary Table 3) was applied for gene prediction. EVidenceModeler (v1.1.1) was used to integrate the gene models predicted by the above approaches to obtain a non-redundant consensus gene set ${ }^{77}$. Gene models were identified as those supported by homeologous genes or transcript evidence or by at least two ab initio methods. High-confidence gene models were further filtered to remove short gene models (less than 50 amino acids) and gene models with homology to sequences in the Repbase (E value $\leq 1 \times 10^{-5}$, 
identity $\geq 30 \%$, coverage $\geq 25 \%$ ). Functional annotations of protein-coding genes were conducted based on Pfam protein domains using InterProScan (v5.24-63.0) ${ }^{78}$.

Divergence time estimation. Synteny among Echinochloa subgenomes and other species (O. sativa, S. bicolor, D. exilis, S. italica, and $P$. hallii) was first identified by pairwise BLASTP (e-value less than 1e-10) and DAGchainer (neighboring genes in a single chain to be no more than 10 genes apart, and the minimum chain length equal to five colinear genes $)^{79}$. The non-synonymous substitution $\left(K_{\mathrm{s}}\right)$ values for syntenic homoeologous gene pairs were calculated by KaKs_calculator in the NG model $^{80}$. The of $K_{\mathrm{s}}$ distribution peaks were used to calculate the divergence time using the formula $T=K_{\mathrm{s}} / 2 r$, where $r$ is the nucleotide substitution rate (here $r=6.5 \times 10^{-9}$ mutations $\times \mathrm{bp}^{-1} \times$ generation $\left.^{-1}\right)^{81}$. We employed the divergence of transposon sequences to infer the polyploidization time in Echinochloa ${ }^{10}$.

Phylogenetic analysis. The occurrence of homeologous exchanges (HEs) in polyploids will affect phylogeny inferences. We identified the candidate HE regions in E. crus-galli genome and genes within HE regions were excluded in phylogeny analysis. About $50 \times$ short reads generated from E. haploclada and E. oryzicola assemblies were mapped against reference genome of E. crus-galli using Bowtie2 with default parameters, respectively ${ }^{73}$. Mapping depthes were calculated for 100 $\mathrm{kb}$ sliding windows across E. crus-galli genome. For subgenomes A and B, the windows with mapping depth more than $30 \times$ from $E$. haploclada reads and less than $20 \times$ from $E$. oryzicola reads were regarded as candidate $\mathrm{HE}$ windows. The same criteria were applied for subgenome C. Neighboring candidate HE windows were merged and finally genes within 24 candidate $\mathrm{HE}$ regions in E. crus-galli were removed in following phylogeny analysis. We built the phylogeny among Echinochloa and other species via three methods. First, considering that $K_{\mathrm{s}}$ indicates genetic divergence, we constructed the phylogeny among Echinochloa and neighboring species according to the pairwise $K_{\mathrm{s}}$ peaks. Second, we used a concatenated matrix of 3557 single-copy orthologs identified by OrthoFinder ${ }^{82}$ to build an ML tree. None of these single-copy genes were within candidate HE regions, which eliminated the noise by HE in the phylogeny inference within Echinochloa. The amino acid sequences were aligned using MAFFT (v7.310) ${ }^{83}$. GBlocks (v0.91b) ${ }^{84}$ was used to trim alignment gaps with the parameter of '-b4 $=5-\mathrm{b} 5=\mathrm{h}$ '. The tree was built by using RaxML ${ }^{85}$ with 1000 bootstrap replications. The substitution model was set as PROTGAMMA $+\mathrm{JTT}+\mathrm{F}$, as recommended by ProtTest ${ }^{86}$ and ModelFinder implemented in IQ-TREE (v1.6.12) ${ }^{87}$. Third, we built a total of 3557 single gene trees individually using IQ-TREE with the best amino acid substitution model and obtained an integrated coalescent-based phylogeny by using ASTRAL (v5.7.4) ${ }^{88}$. To analyze the potential ILS in Echinochloa and introgression among genera, we first counted the numbers of three topologies in each triplet with an outgroup. Subsequently, we employed QuIBL ${ }^{32}$, a method based on internal branch length, to quantify the proportions of ILS and introgression contributions to topology incongruence.

Global collection and phenotyping of Echinochloa plants. A total of 737 Echinochloa samples were collected from 16 rice-producing countries (China, Japan, Korea, Malaysia, India, and Pakistan in Asia; Brazil, US, Costa Rica, Uruguay, and Venezuela in America; Italy, Germany, Portugal, and Russia in Europe; Australia in Oceania). These samples included 328 accessions from Yangtze River basin, China, collected in the previous study ${ }^{6}$ (Supplementary Data 3). These materials (except Brazil samples) were planted and phenotyped in the paddy fields at the China National Rice Research Institute, China, with eight individuals per accession in 2017, 2018, and 2020. The herbicide resistance phenotypes of barnyard grasses were categorized as Brazilian and Italian populations (Supplementary Note 1). The distribution of samples was illustrated using R (v4.0.3, https://www.r-project.org/).

Resequencing and variant calling. DNA was extracted from fresh leaves using a routine protocol. Resequencing paired-end reads were generated using Illumina HiSeq 4000. Raw data were first filtered by NGSQC Toolkit (v2.3.348) ${ }^{89}$. Clean paired-end reads of each accession were then aligned to the updated reference genome of E. crus-galli (STB08) using Bowtie2 with default parameters ${ }^{72}$. An integrated pipeline ${ }^{6}$ was used in calling and filtering whole-genome variants (SNPs and InDels). To eliminate the mapping errors caused by high sequence similarities between subgenomes, the resequencing reads of STB08 were remapped to the reference, and variants were called in the same pipeline. These variants called in STB08 were removed from the final variant dataset. These variants were further filtered with the minor allele frequency (MAF) greater than 0.01 and missing rate less than $30 \%$. For E. colona accessions, the clean reads were remapped to the reference genome of E. colona (PI473783) and variants were called using the same pipeline as mapping to $E$. crus-galli reference genome. The variants with MAF greater than 0.05 and missing rate less than $20 \%$ were further filtered. All variants were annotated by SnpEff (v3.652) ${ }^{90}$.

Population structure and genetic diversity. To determine the species of each accession, read mapping rates to STB08 and genome coverage were calculated using Samtools (v1.5) ${ }^{91}$. FastStructure ${ }^{92}$ was applied to infer the ancestry of all accessions with $K$-values ranging from 2 to 7 , using whole-subgenome SNPs and synonymous SNPs in subgenomes A, B, and C. An approximately ML tree was built using SNPs from both subgenomes A and B for 705 accessions (E. colona was excluded due to low genome coverage) with $E$. walteri as an outgroup by FastTreeMP 93 , with 1000 bootstrap replications. For E. colona phylogeny, we constructed an approximately ML tree using whole-genome SNPs across 32 accessions of $E$. colona with one accession from E. crus-galli as an outgroup with 1000 bootstrap replications. These trees were illustrated in iTOL (http:// itol.embl.de). A principal component analysis was performed using the PLINK software (v1.90b6.20) ${ }^{94}$ with a pruned subset of whole-genome SNPs based on LD (10 SNPs in each 50-kb sliding window with pairwise Pearson's correlation efficient $r^{2}$ less than 0.5$)$. The nucleotide diversity $(\pi)$ of each population was calculated using VCFtools (v0.1.17) ${ }^{95}$ in non-overlapping 20 -kb windows. The average $\pi$ of all sliding windows was regarded as the value at the whole (sub)genome level across different groups or subgenomes.

Demographic history. To recover the divergence history and explore the role of gene flow in the formation of E. crus-galli complex, composite ML inference, based on site frequency spectrum (SFS), was conducted. To calculate the unfolded joint $\mathrm{SFS}^{96,97}$, we used the est-sfs program (v2.03) ${ }^{98}$ to infer the ancestral state for each allele with one individual (PI477956) from E. walteri as an outgroup. The joint SFS was built using only unlinked synonymous SNPs with known ancestral alleles, using the software easySFS.py (https://github.com/isaacovercast/easySFS). The SNP data of each group were down-projected to an SFS with equivalent sampling sizes across groups to decrease the effect of different levels of missing data between groups.

The divergence scenarios for the five E. crus-galli varieties with E. oryzicola var oryzicola as an outgroup were set with a focus on the ancestry of E. crus-galli var. crus-pavonis (whether hybridization or continuous gene flow) (Supplementary Fig. 24). We calculated the likelihood function for different demographic scenarios using fastsimcoal 2 software ${ }^{34}$. For each scenario, 100000 coalescent simulations per likelihood estimation (i.e. -n100000) and 40 expectation-conditional maximization (ECM) cycles (-L40) were used as the command line parameters for each run. The Akaike information criterion (AIC) was used to compare different models. In this case, AIC $=2 k-2 \ln$ (MaxEstLhood), where $k$ is the number of parameters estimated by each model, and MaxEstLhood is the ML function value for each model. Moreover, when searching for an ML value, fastsimcoal 2 may reach a local optimum instead of a global optimum. Thus, we repeated each step at least twice to ensure the searching did not stop in a local optimum, thereby getting better estimates of the global optimum.

The existence and direction of the gene flow between groups were tested using 14 independent scenarios (Supplementary Fig. 25). The best scenario of gene flow for 15 group pairs were then used to set migration matrix for the best scenario of group divergence. The scenario combining divergence history and gene flow were run 10 times to obtain the best parameter estimation. Thereafter, 100 independent DNA polymorphism datasets were simulated as joint SFSs conditional on estimated demographic parameters. An ML analysis was then applied to each joint SFS over 40 ECM cycles to obtain confidence intervals (CIs) for final estimates.

In addition, Stairway Plot $(\mathrm{v} 2.0)^{36}$ was used to infer effective population size change through time in recent thousand years based on both folded and unfolded SFSs. The SFS for each group was generated and folded from the same SNP dataset used for the fastsimcoal2 analysis.

Phylogeny of Echinochloa chloroplast genomes. Taken the chloroplast genome of Panicum virgatum (NC_015990.1) as a seed, the reads which could be used for genome de novo assembly were picked out from clean resequencing paired-end reads using NOVOplasty (v4.2) ${ }^{99}$ and then were aligned to the reference chloroplast genome of E. crus-galli (BTS02; KJ000047). Using the GATK pipeline, a final set of 729 chloroplast SNPs were called across a total of 607 Echinochloa individuals with the filtering parameters of MAF greater than 0.05 and missing rate less than 0.2. An ML tree was built using MEGACC (v10.1.8) ${ }^{100}$ with 1000 bootstrap replications and, the tree was rooted using the chloroplast genome of Setaria italica as an outgroup.

ABBA-BABA statistics. Incongruence between nuclear and chloroplast genome phylogeny revealed chloroplast capture in Echinochloa. We performed ABBA$\mathrm{BABA}$ test to detect the signals of introgression from E. oryzicola var. oryzicola to E. crus-galli var. crus-galli. The test ABBA-BABA or D statistics tested for gene introgression between a triplet of three close populations ' $\mathrm{P} 1$ ', ' $\mathrm{P} 2$ ', and ' $\mathrm{P} 3$ ', using an outgroup 'O' following the topology (((P1, P2), P3), O) (Supplementary Fig. 28a). In our analysis, four topologies were tested, with E. walteri or E. oryzicola var. hainanensis as an outgroup (Supplementary Fig. 28b). The $f_{\mathrm{d}}$ statistics was calculated in sliding windows with a window size of $100 \mathrm{~kb}$ and a step size of $20 \mathrm{~kb}$, using the python scripts available at https://github.com/simonhmartin/ genomics_general. According to the filtering criteria used by Zhou et al. ${ }^{37}$, we converted the $f_{\mathrm{d}}$ values to zero, when they were less than 0 or greater than 1 . In summary, PGI (Proportion of Genomic Introgression) was used to quantify the overall introgression extent across the whole genome ${ }^{37}$.

Local adaptation analysis. Population fixation statistics $\left(F_{\mathrm{st}}\right)$ were estimated using VCFtools in 20-kb sliding windows between E. crus-galli var. crus-galli and E. crus-galli var. praticola and between high-latitude and low-latitude groups in 
E. oryzicola var. oryzicola. Genes in highly differentiated blocks (top 5\% windows in $F_{\text {st }}$ ) were annotated by rice function-validated orthologs (Supplementary Data 5 and 6). Neutral test (Tajima's $D$ ) was calculated by VCFtools in $20-\mathrm{kb}$ windows in each population. The GWAS for the FT of 461 individuals of E. crus-galli in 2017 was conducted using mixed linear modeling implemented in EMMAX ${ }^{101}$. The genome-wide significance thresholds were set as a Bonferroni correction of $\alpha=0.05$. The haplotype analyses of $H d 1$ and $N R T 1.1 B$ were carried using custom scripts. The Fisher's exact test was used to calculate significance in the GO enrichment analysis.

Herbicide resistance analyses. The genes encoding herbicide-target enzymes ALS, EPSPS, ACCase, and psbA in Echinochloa genomes were identified by BLASTP. The Echinochloa protein sequences of these enzymes were aligned with the corresponding sequences from the other species (O. sativa and S. italica) using MAFFT. We determined the atlas of causative mutations in global barnyard grass. The herbicide resistance of accessions sampled from Brazil and Italy were phenotyped (Supplementary Data 9 and Supplementary Note 5). For accessions with no causative mutations but herbicide resistance phenotype in a Brazilian population, we explored the possibility of gene copy number alterations underlying resistance by measuring the gene family sizes inducing non-target-sites resistance (cytochrome P450 monooxygenase, GST, and ABC transporter).

Genomic features of barnyard millet domestication. The genetic diversity $(\pi)$ and Tajima's $D$ of barnyard millet and grass and genomic differentiation $\left(F_{\mathrm{st}}\right)$ between them were estimated using VCFtools in 20-kb windows. PopLDdecay (v3.31; https://github.com/BGI-shenzhen/PopLDdecay) was used to calculate the linkage disequilibrium (LD) on variant pairs within a 300-kb window. LD decay was estimated using the distance where the Pearson's correlation efficient $\left(r^{2}\right)$ decreased to half of its maximum value.

Detection of CNV of the NB-ARC gene family. Pipeline CNVings (Copy Number Variation inferred from next-generation sequencing) was used to compare gene family copy number differences at the population level using the NGS resequencing data (See details in Supplementary Note 6). CNVings estimated the copy number of NB-ARC gene family by measuring the relative abundance compared to the reference genome. The physical positions of all NB-ARC genes in the reference genome were annotated by InterProScan $(e$-value $<1 \mathrm{e}-10)$. The abundance of NB-ARC gene family across the whole genome was measured by the sum of the average depths of all NB-ARC loci using Mosdepth (v0.2.9 ${ }^{102}$. The copy number was then standardized by the average mapping depth across the whole genome. Five platinum-standard rice genomes were used to test the reliability of this pipeline (Supplementary Note 6). Whole-genome resequencing Illumina paired-end reads of wild and cultivated accessions in rice, sorghum, coix, and barnyard grass were used to quantify the size change of the NB-ARC gene family caused by domestication (Supplementary Note 6). Accessions with sequencing depth less than $10 \times$ were removed.

Detection of genes showing high differentiation between weeds and millets. Key genes underlying crucial domestication syndromes (e.g., shattering, plant architecture, and grain size) were identified in E. crus-galli and E. colona genomes by BLASTP to rice genes with an $e$-value less than $1 \mathrm{e}-30$ and identity greater than $50 \%$. The domains were further checked using InterProScan. In rice, shatteringrelated genes, including SH1, sh-h, sh4, qSH1, NPC1, GRF4, SSH1, SH5, and SHAT1, function in the development of the abscission zone of spikelets, whereas genes regulating the alteration of plant architecture, including LA1, LPA1, PROG1, WOX6, WOX11, Hsfa2d, PID, and BRXL4, mainly function in the gravity response pathway mediated by auxin asymmetric distribution. Grain size and weight are regulated by multiple genes, including GS3, GS5, GS6, GW5, GW6, GW7, TGW6, $q G W 8$, GSK2, and other important genes (www.ricedata.cn). Non-synonymous variations with allele frequency difference greater than 0.6 between cultivated and weedy populations were regarded as candidate variations selected in domestication (Supplementary Data 11). Genome-wide differentiation between E. crus-galli var. praticola and var. esculenta, var. oryzoides, between wild and cultivated E. colona, was scanned by $F_{\mathrm{st}}$. The genes within top $5 \%$ differentiated genomic regions were listed in Supplementary Data 12-14.

Reporting summary. Further information on research design is available in the Nature Research Reporting Summary linked to this article.

\section{Data availability}

The Echinochloa genome assemblies and annotations generated in this study are available at National Genomics Data Center (NGDC) database (https://bigd.big.ac.cn) under the accession number PRJCA003883. The HiFi sequencing, Hi-C, and RNA-Seq data for genome assembly and annotation in this study have been deposited in NGDC (accession number PRJCA003883). The raw data of 409 newly re-sequenced individuals are available at NGDC (project accession PRJCA003883). The data of 328 previously resequenced barnyard grass accessions were retrieved from NGDC (accession number PRJCA001519). Besides Echinochloa genomes, other 36 monocot genomes used in this study were downloaded from EnsemblPlants (https://plants.ensembl.org) or National Center for Biotechnology Information (NCBI) GenBank (https://www.ncbi.nlm.nih.gov/) (Supplementary Data 1). The resequencing data of rice, sorghum, and Job's tears were downloaded from the European Nucleotide Archive (ENA) (https://www.ebi.ac.uk/ena/ browser/home) (Supplementary Data 10). Source data are provided with this paper.

\section{Code availability}

The custom scripts used in this study have been deposited in the GitHub repository [https://github.com/bioinplant/Echinochloa_genome].

Received: 15 September 2021; Accepted: 20 January 2022; Published online: 03 February 2022

\section{References}

1. Sharma, G., Barney, J. N., Westwood, J. H. \& Haak, D. C. Into the weeds: new insights in plant stress. Trends Plant Sci. 26, 1050-1060 (2021).

2. Kraehmer, H., Jabran, K., Mennan, H. \& Chauhan, B. S. Global distribution of rice weeds-a review. Crop Prot. 80, 73-86 (2016).

3. Beckie, H. J. \& Tardif, F. J. Herbicide cross resistance in weeds. Crop Prot. 35 , 15-28 (2012).

4. Qiu, J. et al. Genomic variation associated with local adaptation of weedy rice during de-domestication. Nat. Commun. 8, 15323 (2017).

5. Zhang, Y. et al. Effects of different sub-soiling frequencies incorporated into no-tillage systems on soil properties and crop yield in dryland wheat-maize rotation system. Field Crops Res. 209, 151-158 (2017).

6. Ye, C.-Y. et al. Genomic evidence of human selection on Vavilovian mimicry. Nat. Ecol. Evol. 3, 1474-1482 (2019).

7. Renganathan, V. G., Vanniarajan, C., Karthikeyan, A. \& Ramalingam, J. Barnyard millet for food and nutritional security: current status and future research direction. Front. Genet. 11, 500 (2020).

8. Bajwa, A. A. et al. Eco-biology and management of Echinochloa crus-galli. Crop Prot. 75, 151-162 (2015).

9. Guo, L. et al. Echinochloa crus-galli genome analysis provides insight into its adaptation and invasiveness as a weed. Nat. Commun. 8, 1031 (2017).

10. Ye, C.-Y. et al. The genomes of the allohexaploid Echinochloa crus-galli and its progenitors provide insights into polyploidization-driven adaptation. Mol. Plant 13, 1298-1310 (2020).

11. Yu, Q. \& Powles, S. Metabolism-based herbicide resistance and crossresistance in crop weeds: a threat to herbicide sustainability and global crop production. Plant Physiol. 166, 1106-1118 (2014).

12. Fang, J.-B. et al. Formation of (2R)- and (2S)-8-prenylnaringenin glucuronides by human UDP-glucuronosyltransferases. J. Agric. Food Chem. 67, 11650-11656 (2019).

13. Heap, I. The international survey of herbicide resistant weeds. online. http:// www.weedscience.org/Home.aspx (2021).

14. Vázquez-García, J. G. et al. Distribution of glyphosate-resistance in Echinochloa crus-galli across agriculture areas in the Iberian Peninsula. Front. Plant Sci. 12, 617040 (2021).

15. Michael, P. The taxonomy and distribution of Echinochloa species (barnyard grasses) in the Asian-Pacific region, with a review of pertinent biological studies. In: Proceedings of the 18th APWSS Conference (Beijing, China, 28 May-2 June 2001). 57-66 (Standard Press of China, Beijing, 2001).

16. Aoki, D. \& Yamaguchi, H. Genetic relationship between Echinochloa crus-galli and Echinochloa oryzicola accessions inferred from internal transcribed spacer and chloroplast DNA sequences. Weed Biol. Manag. 8, 233-242 (2008).

17. Ye, C.-Y. \& Fan, L. Orphan crops and their wild relatives in the genomic era. Mol. Plant 14, 27-39 (2021)

18. Dewet, J. M. J., Rao, K. E. P., Mengesha, M. H. \& Brink, D. E. Domestication of Sawa millet (Echinochloa colona). Econ. Bot. 37, 283-291 (1983).

19. Yabuno, T. Japanese barnyard millet (Echinochloa utilis, Poaceae) in Japan. Econ. Bot. 41, 484-493 (1987).

20. Yamaguchi, H., Utano, A., Yasuda, K., Yano, A. \& Soejima, A. A molecular phylogeny of wild and cultivated Echinochloa in East Asia inferred from non-coding region sequences of trnT-L-F. Weed Biol. Manag. 5, 210-218 (2005).

21. Yabuno, T. Cytotaxonomic studies on the two cultivated species and the wild relatives in the genus Echinochloa. Cytologia 27, 296-305 (1962).

22. Pellicer, J. \& Leitch, I. J. The plant DNA C-values database (release 7.1): an updated online repository of plant genome size data for comparative studies. New Phytol. 226, 301-305 (2020).

23. Abrouk, M. et al. Fonio millet genome unlocks African orphan crop diversity for agriculture in a changing climate. Nat. Commun. 11, 4488 (2020).

24. VanBuren, R. et al. Single-molecule sequencing of the desiccation-tolerant grass Oropetium thomaeum. Nature 527, 508-511 (2015). 
25. Brown, J. K. M. \& Rant, J. C. Fitness costs and trade-offs of disease resistance and their consequences for breeding arable crops. Plant Pathol. 62, 83-95 (2013).

26. Moghaddam, S. M. et al. The tepary bean genome provides insight into evolution and domestication under heat stress. Nat. Commun. 12, 2638 (2021).

27. Stein, J. C. et al. Genomes of 13 domesticated and wild rice relatives highlight genetic conservation, turnover and innovation across the genus Oryza. Nat. Genet. 50, 285-296 (2018).

28. Lisch, D. How important are transposons for plant evolution? Nat. Rev. Genet. 14, 49-61 (2013)

29. Lam, K. C., Ibrahim, R. K., Behdad, B. \& Dayanandan, S. Structure, function, and evolution of plant O-methyltransferases. Genome 50, 1001-1013 (2007).

30. Kumar, R. et al. Evolutionary profiling of group II pyridoxal-phosphatedependent decarboxylases suggests expansion and functional diversification of histidine decarboxylases in tomato. Plant Genome 9, 1-15 (2016).

31. Saarela, J. M. et al. A 250 plastome phylogeny of the grass family (Poaceae): topological support under different data partitions. PeerJ 6, e4299 (2018).

32. Edelman, N. B. et al. Genomic architecture and introgression shape a butterfly radiation. Science 366, 594 (2019).

33. Zou, X.-H. et al. Analysis of 142 genes resolves the rapid diversification of the rice genus. Genome Biol. 9, R49 (2008).

34. Excoffier, L., Dupanloup, I., Huerta-Sanchez, E., Sousa, V. C. \& Foll, M. Robust demographic inference from genomic and SNP data. PLoS Genet. 9, e1003905 (2013).

35. Yabuno, T. Cytological relationship between Echinochloa oryzicola Vasing. and the French strain of E. phyllopogon Stapf subsp. oryzicola (Vasing.) Koss. Cytologia 46, 393-396 (1981)

36. Liu, X. \& Fu, Y.-X. Stairway Plot 2: demographic history inference with folded SNP frequency spectra. Genome Biol. 21, 280 (2020).

37. Zhou, Y. et al. Triticum population sequencing provides insights into wheat adaptation. Nat. Genet. 52, 1412-1422 (2020).

38. Walkowiak, S. et al. Multiple wheat genomes reveal global variation in modern breeding. Nature 588, 277-283 (2020).

39. Bock, R. The give-and-take of DNA: horizontal gene transfer in plants. Trends Plant Sci. 15, 11-22 (2010).

40. Gaut, B. S., Miller, A. J. \& Seymour, D. K. Living with two genomes: grafting and its implications for plant genome-to-genome interactions, phenotypic variation, and evolution. Annu. Rev. Genet. 53, 195-215 (2019).

41. $\mathrm{Hu}, \mathrm{B}$. et al. Variation in NRT1.1B contributes to nitrate-use divergence between rice subspecies. Nat. Genet. 47, 834-838 (2015).

42. Yu, Q. \& Powles, S. B. Resistance to AHAS inhibitor herbicides: current understanding. Pest Manag. Sci. 70, 1340-1350 (2014).

43. Murphy, B. P. \& Tranel, P. J. Target-site mutations conferring herbicide resistance. Plants-Basel 8, 382 (2019).

44. Yang, X. et al. Exploring quinclorac resistance mechanisms in Echinochloa crus-pavonis from China. Pest Manag. Sci. 77, 194-201 (2021).

45. LeClere, S., Wu, C., Westra, P. \& Sammons, R. D. Cross-resistance to dicamba, 2,4-D, and fluroxypyr in Kochia scoparia is endowed by a mutation in an AUX/IAA gene. Proc. Natl Acad. Sci. USA 115, 2911-2920 (2018).

46. De Figueiredo, M. R. A. et al. An in-frame deletion mutation in the degron tail of auxin co-receptor IAA2 confers resistance to the herbicide 2,4-D in Sisymbrium orientale. bioRxiv. Preprint at https://doi.org/10.1101/ 2021.03.04.433944 (2021).

47. Powles, S. B. \& Yu, Q. Evolution in action: plants resistant to herbicides. Annu. Rev. Plant Biol. 61, 317-347 (2010).

48. Gaines, T. A. et al. Mechanisms of evolved herbicide resistance. J. Biol. Chem. 295, 10307-10330 (2020).

49. Patterson, E. L., Pettinga, D. J., Ravet, K., Neve, P. \& Gaines, T. A. Glyphosate resistance and EPSPS gene duplication: convergent evolution in multiple plant species. J. Hered. 109, 117-125 (2018).

50. Pan, L. et al. Aldo-keto reductase metabolizes glyphosate and confers glyphosate resistance in Echinochloa colona. Plant Physiol. 181, 1519-1534 (2019).

51. Pan, L. et al. An ABCC-type transporter endowing glyphosate resistance in plants. Proc. Natl Acad. Sci. USA 118, e2100136118 (2021).

52. Yabuno, T. A note on barnyard millet. SABRAO Newsl. 3, 43-45 (1971).

53. Meyer, R. S. \& Purugganan, M. D. Evolution of crop species: genetics of domestication and diversification. Nat. Rev. Genet. 14, 840-852 (2013).

54. Soltis, P. S., Marchant, D. B., Van de Peer, Y. \& Soltis, P. S. Polyploidy and genome evolution in plants. Curr. Opin. Genet. Dev. 35, 119-125 (2005).

55. Van de Peer, Y., Mizrachi, E. \& Marchal, K. The evolutionary significance of polyploidy. Nat. Rev. Genet. 18, 411-424 (2017).

56. Appels, R. et al. Shifting the limits in wheat research and breeding using a fully annotated reference genome. Science 361, 7191 (2018).

57. Sun, Y., Shang, L., Zhu, Q.-H., Fan, L. \& Guo, L. Twenty years of plant genome sequencing: achievements and challenges. Trends Plant Sci. 1360-1385 (2021).
58. Rozhevits, R. Y. Grasses: an introduction to the study of fodder and cereal grasses (Indian National Scientific Documentation Centre, New Delhi, 1937).

59. Guo, Z.-H. et al. Genome sequences provide insights into the reticulate origin and unique traits of woody bamboos. Mol. Plant 12, 1353-1365 (2019).

60. Yu, Q. \& Powles, S. Metabolism-based herbicide resistance and crossresistance in crop weeds: a threat to herbicide sustainability and global crop production. Plant Physiol. 166, 1106-1118 (2014).

61. Kreiner, J. M., Stinchcombe, J. R. \& Wright, S. I. Population genomics of herbicide resistance: adaptation via evolutionary rescue. Annu. Rev. Plant Biol. 69, 611-635 (2018)

62. Kreiner, J. M. et al. Multiple modes of convergent adaptation in the spread of glyphosate-resistant Amaranthus tuberculatus. Proc. Natl Acad. Sci. USA 116, 21076-21084 (2019)

63. $\mathrm{Yu}, \mathrm{H}$. et al. A route to de novo domestication of wild allotetraploid rice. Cell 184, 1156-1170 (2021).

64. Cheng, H., Concepcion, G. T., Feng, X., Zhang, H. \& Li, H. Haplotyperesolved de novo assembly using phased assembly graphs with hifiasm. Nat. Methods 18, 170 (2021)

65. Li, H. Minimap2: pairwise alignment for nucleotide sequences. Bioinformatics 34, 3094-3100 (2018)

66. Vaser, R., Sovic, I., Nagarajan, N. \& Sikic, M. Fast and accurate de novo genome assembly from long uncorrected reads. Genome Res. 27, 737-746 (2017).

67. Wu, T. D. \& Watanabe, C. K. GMAP: a genomic mapping and alignment program for mRNA and EST sequences. Bioinformatics 21, 1859-1875 (2005)

68. Li, H. \& Durbin, R. Fast and accurate short read alignment with BurrowsWheeler transform. Bioinformatics 25, 1754-1760 (2009).

69. Zhang, X., Zhang, S., Zhao, Q., Ming, R. \& Tang, H. Assembly of allele-aware, chromosomal-scale autopolyploid genomes based on Hi-C data. Nat. Plants $\mathbf{5}$, 833-845 (2019).

70. Simao, F. A., Waterhouse, R. M., Ioannidis, P., Kriventseva, E. V. \& Zdobnov, E. M. BUSCO: assessing genome assembly and annotation completeness with single-copy orthologs. Bioinformatics 31, 3210-3212 (2015).

71. Ou, S., Chen, J. \& Jiang, N. Assessing genome assembly quality using the LTR Assembly Index (LAI). Nucleic Acids Res. 46, e126 (2018).

72. Langmead, B. \& Salzberg, S. L. Fast gapped-read alignment with Bowtie 2. Nat Methods 9, 357-359 (2012).

73. Marcais, G. \& Kingsford, C. A fast, lock-free approach for efficient parallel counting of occurrences of k-mers. Bioinformatics 27, 764-770 (2011).

74. Benson, G. Tandem repeats finder: a program to analyze DNA sequences. Nucleic Acids Res. 27, 573-580 (1999).

75. Salamov, A. A. \& Solovyev, V. V. Ab initio gene finding in Drosophila genomic DNA. Genome Res. 10, 516-522 (2000).

76. Stanke, M. et al. AUGUSTUS: ab initio prediction of alternative transcripts. Nucleic Acids Res. 34, W435-W439 (2006).

77. Haas, B. J. et al. Automated eukaryotic gene structure annotation using EVidenceModeler and the program to assemble spliced alignments. Genome Biol. 9, R7 (2008).

78. Zdobnov, E. M. \& Apweiler, R. InterProScan-an integration platform for the signature-recognition methods in InterPro. Bioinformatics 17, 847-848 (2001).

79. Haas, B. J., Delcher, A. L., Wortman, J. R. \& Salzberg, S. L. DAGchainer: a tool for mining segmental genome duplications and synteny. Bioinformatics 20, 3643-3646 (2004)

80. Zhang, Z. et al. KaKs_calculator: calculating Ka and Ks through model selection and model averaging. Genom. Proteom. Bioinf. 4, 259-263 (2006).

81. Molina, J. et al. Molecular evidence for a single evolutionary origin of domesticated rice. Proc. Natl Acad. Sci. USA 108, 8351-8356 (2011).

82. Emms, D. M. \& Kelly, S. OrthoFinder: phylogenetic orthology inference for comparative genomics. Genome Biol. 20, 238 (2019).

83. Katoh, K. \& Standley, D. M. MAFFT multiple sequence alignment software version 7: improvements in performance and usability. Mol. Biol. Evol. 30 772-780 (2013).

84. Castresana, J. Selection of conserved blocks from multiple alignments for their use in phylogenetic analysis. Mol. Biol. Evol. 17, 540-552 (2000).

85. Stamatakis, A. RAxML version 8: a tool for phylogenetic analysis and postanalysis of large phylogenies. Bioinformatics 30, 1312-1313 (2014)

86. Darriba, D. et al. ProtTest 3: fast selection of best-fit models of protein evolution. Bioinformatics 27, 1164-1165 (2011).

87. Nguyen, L.-T., Schmidt, H. A., von Haeseler, A. \& Bui Quang, M. IQ-TREE: a fast and effective stochastic algorithm for estimating maximum-likelihood phylogenies. Mol. Biol. Evol. 32, 268-274 (2015).

88. Zhang, C., Rabiee, M., Sayyari, E. \& Mirarab, S. ASTRAL-III: polynomial time species tree reconstruction from partially resolved gene trees. BMC Bioinformatics 19, 847-848 (2018).

89. Patel, R. K. \& Jain, M. NGS QC Toolkit: a toolkit for quality control of next generation sequencing data. PLoS ONE 7, e30619 (2012). 
90. Cingolani, P. et al. A program for annotating and predicting the effects of single nucleotide polymorphisms, SnpEff: SNPs in the genome of Drosophila melanogaster strain w(1118); iso-2; iso-3. Fly 6, 80-92 (2012).

91. Li, H. et al. The Sequence Alignment/Map format and SAMtools. Bioinformatics 25, 2078-2079 (2009).

92. Raj, A., Stephens, M. \& Pritchard, J. K. fastSTRUCTURE: variational inference of population structure in large SNP data sets. Genetics 197, 573-U207 (2014).

93. Price, M. N., Dehal, P. S. \& Arkin, A. P. FastTree: computing large minimum evolution trees with profiles instead of a distance matrix. Mol. Biol. Evol. 26, 1641-1650 (2009).

94. Chang, C. C. et al. Second-generation PLINK: rising to the challenge of larger and richer datasets. GigaScience 4, 7 (2015).

95. Danecek, P. et al. The variant call format and VCFtools. Bioinformatics 27, 2156-2158 (2011).

96. Li, H. \& Stephan, W. Inferring the demographic history and rate of adaptive substitution in Drosophila. PLoS Genet. 2, 1580-1589 (2006).

97. Gutenkunst, R. N., Hernandez, R. D., Williamson, S. H. \& Bustamante, C. D. Inferring the joint demographic history of multiple populations from multidimensional SNP frequency data. PLoS Genet. 5, e1000695 (2009).

98. Keightley, P. D. \& Jackson, B. C. Inferring the probability of the derived vs. the ancestral allelic state at a polymorphic site. Genetics 209, 897-906 (2018)

99. Dierckxsens, N., Mardulyn, P. \& Smits, G. NOVOPlasty: de novo assembly of organelle genomes from whole genome data. Nucleic Acids Res. 45, 18 (2017).

100. Kumar, S., Stecher, G., Li, M., Knyaz, C. \& Tamura, K. MEGA X: molecular evolutionary genetics analysis across computing platforms. Mol. Biol. Evol. 35, 1547-1549 (2018).

101. Kang, H. M. et al. Variance component model to account for sample structure in genome-wide association studies. Nat. Genet. 42, 348-354 (2010).

102. Pedersen, B. S. \& Quinlan, A. R. Mosdepth: quick coverage calculation for genomes and exomes. Bioinformatics 34, 867-868 (2018).

\section{Acknowledgements}

This project was supported by the Department of Science and Technology of Zhejiang Province (2022C02032 and 2020C02002), the National Natural Science Foundation (32170621 and 31901899), Zhejiang Natural Science Foundation (LZ17C130001), Jiangsu Collaborative Innovation Center for Modern Crop Production and 111 Project (B17039) to L.F. We thank Yongfeng Li (Institute of Plant Protection, Jiangsu Academy of Agricultural Sciences, China) for help with material collection and Prof. Ya-Long Guo (Institute of Botany, Chinese Academy of Sciences, China) for his insightful suggestions.

\section{Author contributions}

L.F. and C.-Y.Y. designed and supervised the study. W.T., A.M.J., L.S., K.M.O., B.-K.S., T.-S.C, D.W., H.Y., and C.-Y.Y. collected samples. W.T., C.-Y.Y., D.W., L.J., B.J., X.W.,
C.D., and L.G. phenotyped samples and prepared materials for sequencing. L.C., A.M.J., and L.S. conducted the herbicide resistance experiments. D.W., L.J., B.J., and C.D. performed genome assembly and annotation. D.W., E.S., C.-Y.Y., Y.F., S.L., H.-Y.L., F.L., B.J., L.X., H.X., and Q.Q. performed comparative genomic and population genetics analyses. D.W., L.F., C.-Y.Y., Q.-H.Z., and Q.Y. wrote the manuscript. K.M.O., Y.Q., H.Y., M.P.T., H.C., S.D. and J.Q. discussed the results and edited the manuscript. All authors read and approved the manuscript.

\section{Competing interests}

The authors declare no competing interests.

\section{Additional information}

Supplementary information The online version contains supplementary material available at https://doi.org/10.1038/s41467-022-28359-9.

Correspondence and requests for materials should be addressed to Chu-Yu Ye.

Peer review information Nature Communications thanks Simon Krattinger, Eric Patterson, Jue Ruan and the other, anonymous, reviewer(s) for their contribution to the peer review of this work. Peer reviewer reports are available.

Reprints and permission information is available at http://www.nature.com/reprints

Publisher's note Springer Nature remains neutral with regard to jurisdictional claims in published maps and institutional affiliations.

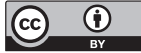

Open Access This article is licensed under a Creative Commons Attribution 4.0 International License, which permits use, sharing, adaptation, distribution and reproduction in any medium or format, as long as you give appropriate credit to the original author(s) and the source, provide a link to the Creative Commons license, and indicate if changes were made. The images or other third party material in this article are included in the article's Creative Commons license, unless indicated otherwise in a credit line to the material. If material is not included in the article's Creative Commons license and your intended use is not permitted by statutory regulation or exceeds the permitted use, you will need to obtain permission directly from the copyright holder. To view a copy of this license, visit http://creativecommons.org/ licenses/by/4.0/.

(C) The Author(s) 2022 